\title{
A mesoscopic model for large-scale simulation of pedestrian dynamics
}

\author{
Antoine Tordeux ${ }^{1, *}$, Gregor Lämmel ${ }^{2}$, Flurin S. Hänseler ${ }^{3}$ and Bernhard Steffen ${ }^{4}$ \\ ${ }^{1}$ University of Wuppertal, Germany \\ ${ }^{3}$ Delft University of Technology, The Netherlands \\ ${ }^{2}$ German Aerospace Center, Germany \\ ${ }^{4}$ Forschungszentrum Jülich, Germany
}

\begin{abstract}
A mesoscopic pedestrian model is proposed, considering pedestrians as individuals and describing their movement by means of aggregate density-flow relationships. The model builds on a stochastic process, describing transition rates among adjacent sites on a lattice. Each lattice can contain several pedestrians. The approach is minimal and fast to simulate, and, by construction, capable of capturing population heterogeneity as well as variability in walking behaviour and en-route path choice. The model is more efficient than microscopic models, and potentially more accurate than macroscopic ones. We calibrate and validate the model using real data and carry out several numerical experiments to present its key properties and possible applications for simulation of large-scale scenarios.
\end{abstract}

Keywords: Pedestrian dynamics, mesoscopic model, large-scale simulation

\section{Introduction}

Pedestrian dynamics have attracted the attention of scientists and practitioners for several decades (Fruin; 1971; Hankin and Wright; 1958). Recent efforts testify to their increasing importance, fuelled by a rapidly growing world population and an advancing urbanisation.

While there is a general need to better understand pedestrian dynamics as such, there is a particular need for adequate models that can be used to improve the safety, efficiency and comfort of pedestrian facilities. In the past, such models have been successfully deployed for studying pilgrim movements on holy sites (AlGadhi and Mahmassani; 1991), crowd behaviour in large-scale events including panic (Helbing et al.; 2000a, 2002), evacuation at the level of buildings or entire regions (Kirchner and Schadschneider; 2002; Lämmel et al.; 2010), as well as pedestrian flows occurring in transportation hubs (Daamen; 2004; Hänseler; 2016) or in urban facilities (Borgers and Timmermans; 1986; Hoogendoorn and Bovy; 2004).

Despite these advancements, there is still a lack of models that are at the same time accurate and efficient (Duives et al.; 2013). Among models that have made it to the engineering practice, there is a clear dominance of microscopic models, such as the social force model (Helbing and Molnár; 1995). Microscopic models consider pedestrians as a set of individuals, whose movement through space and time is described in detail. Their strengths include the ability to describe heterogeneity across individuals, their capability to reproduce collective phenomena such as lane formation, as well as their realism and visual appeal (Helbing et al.; 2001). On the other hand, microscopic models are computationally expensive, and difficult to calibrate on real data (Hoogendoorn and Daamen; 2009).

Large-scale or time-critical applications, for which computational efficiency and a stringent validation are key, have thus not yet fully benefited from the advancements in pedestrian modelling. Notably, this includes large-scale planning, real-time monitoring of crowds, as well as crowd management, where pedestrians are actively controlled (Abdelghany et al.; 2012; Seer et al.; 2008; Shende et al.; 2011).

In principle, macroscopic models may be used in that context, considering pedestrians as a continuum (Hughes; 2002). Macroscopic models typically rely on a small set of parameters that are accessible to physical interpretation, can be readily calibrated on data, and often run orders of magnitude faster than real-time. However, macroscopic models have limitations as well. Most importantly, they ignore differences in walking characteristics among pedestrians. Such heterogeneity may be due to differences in trip purpose, cultural background, age, health or other factors that are known to influence walking behaviour (Seyfried et al.; 2005; Weidmann; 1992). Related to that, most macroscopic models are deterministic in nature, and reproducing natural variability in densities or walking times, as it may be required for level-of-service assessment or demand estimation (Hänseler, Molyneaux and Bierlaire; 2017), may be difficult.

*Email: tordeux@uni-wuppertal.de 
In this contribution, we put forward a new type of pedestrian flow model that may be referred to as mesoscopic. The proposed model provides an interesting compromise between the level of detail of the flow description and computational complexity for the simulation of large scale scenarios, e.g., evacuation at the level of buildings or entire regions as well as pedestrian flows occurring in transportation hubs or in urban facilities. An explicit representation of pedestrians allows to consider individual differences in walking behaviour, for instance in terms of free-flow speeds. It is inherently stochastic, and thus able to reproduce 'natural' fluctuations in walking speeds or en-route path choice. The propagation of pedestrians is described at the aggregate level, thus allowing for low computational cost. In fact, it may be even more efficient than discrete-time macroscopic models under certain conditions. Finally, the proposed model can reproduce several well-known features of pedestrian traffic such as the occurrence of a fundamental diagram and its variability, queuing and rarefaction, or lane formation in counter-flow, and provides good estimates of travel or egress times.

The remainder of this article is structured as follows: Sec. 2 provides a brief review of existing pedestrian flow models. An analytical and a simulation-based formulation of the model are given in Sec. 3, while its properties are analysed by simulation in Sec. 4. Some comparison to real data is carried out in Sec. 5. Sec. 6 provides a large-scale numerical example and Sec. 7 concludes with final remarks.

\section{Literature review}

Some of the key approaches to modelling pedestrian flow at the micro-, macro- and mesoscopic level are described in the following. Related models from physics and vehicular traffic, forming the foundation of the approach presented in this manuscript, are also briefly exposed.

In microscopic models, collective walking phenomena emerge from mutual interactions among individuals. In the aforementioned social force model, pedestrians are subject to Newton-like forces, and the dynamics are governed by equations of motion (Helbing and Molnár; 1995). It represents by and large the most popular pedestrian walking model, owing to its accuracy and visual appeal that make up for its relatively high computational complexity. Cellular automata describe the local movement of pedestrians by a set of behavioural rules that determine the transition probabilities to adjacent locations (Blue and Adler; 2001). These transition probabilities depend on the desired walking direction of a pedestrian, and the availability of space in the vicinity. Burstedde et al. (2001) additionally consider the notion of a floor field to steer pedestrians along their desired path, managing to describe collective effects such as lane formation. The resulting models are known for their low computational cost, but also for their limited capability to consider population heterogeneity, or to reproduce plausible pedestrian dynamics in multi-directional or congested flow. More recent models focus on the reproduction of plausible density-speed relationships, but still lack a realistic representation of heterogeneity (Crociani and Lämmel; 2016; Flötteröd and Lämmel; 2015). A next-step model based on a discrete choice framework is proposed by Antonini et al. (2006). Their approach focuses on the short-term behaviour of individuals as a response to the presence of other pedestrians, and a particular effort to obtain a reliable model calibration is made. Last but not least, in the gaming industry collision-avoidance models have gained a certain popularity due to their visual realism. Such models rely on predictive measures, or on synthetic vision, to anticipate and prevent collisions (Ondřej et al.; 2010; Paris et al.; 2007).

Macroscopic models consider pedestrians as a 'thinking fluid,' and have gained increasing popularity since a continuum theory for pedestrian flow has first been proposed by Hughes (2002). Typically, a conservation equation is combined with a phenomenological density-flow relationship, widely referred to as 'fundamental diagram.' To model the walking direction, a continuous floor field is assumed that leads pedestrians to their desired destination, while minimising travel time and avoiding areas of high density. Several researchers have proposed extensions to the model, or developed numerical schemes for its efficient computation (Hänseler et al.; 2014; Huang et al.; 2009). Colombo and Rosini (2005) consider 'over-compressed' densities that may arise in situations of panic, providing a mathematical framework for studying non-classical shocks in one-dimensional space. Another extension is due to Hänseler, Lam, Bierlaire, Lederrey and Nikolić (2017), who study anisotropic flow, i.e., the circumstance that walking speed depends on the walking direction in multi-directional flow at intermediate and high densities. Using a measure-theoretic approach, Piccoli and Tosin (2011) describe a model based on a generalised continuity equation in which a family of measures is pushed forward by a flow map. Finally, Hoogendoorn et al. (2014) derive a continuum model from Helbing's social force model by assuming a homogeneous population. All acceleration terms are set to zero, yielding an equilibrium model that is similar to the approach proposed by Hughes (2002).

Mesoscopic models consider pedestrians as individuals, but describe their propagation by aggregate relationships. They are better at incorporating and providing population heterogeneity than macroscopic models, 
and typically more efficient than microscopic models. Løvås (1994) proposes a network-based pedestrian flow model involving a queueing system. Pedestrian facilities are modelled as a network of walkway sections, where each pedestrian is treated as a separate flow object that interacts with others. To describe the propagation of walking pedestrians, an empirical density-speed relationship is used. The model is particularly useful for situations where a network representation readily follows from the building geometry, such as for longitudinal corridors. For general building layouts, as well as for situations involving multi-directional flow, the model is less practical. Another example is due to Treuille et al. (2006), who transform Hughes' continuum model into a particle representation. Despite the model being based on continuum dynamics, some explicit particle-to-particle interaction is considered, for instance to enforce a pair-wise minimum distance between pedestrians. The main purpose of this model lies in the provision of real-time animations, which for most applications in transportation engineering is less relevant. Finally, while not being mesoscopic in the strict sense, hybrid models may be mentioned. Hybrid models combine models formulated at different levels of aggregation to yield a compromise between computational complexity and accuracy (Crociani et al.; 2016; Lämmel et al.; 2014). Ensuring consistency across levels, both in terms of parameter calibration and in terms of state variables, makes using such models however often difficult.

Two recent vehicular models may finally be discussed. Both of them rely on a stochastic misanthrope process, like the model proposed in this work. The misanthrope process has originally been introduced by Cocozza-Thivent (1985) to study electrolytes. It represents a jump process describing particles evolving on a lattice of finite dimension, where the transition rates depend on the states of the departure and arrival site. More precisely, the term misanthrope reflects the property that the transition rate is a non-decreasing function of the particle number on the departure site, and a non-increasing function of the particle number on the arrival site. In other words, the demand of movement within a space section increases as the density increases, while the supply of a section decreases as the density increases. We note that in the context of proxemics (Hall et al.; 1968), this mechanism can be behaviourally interpreted as the tendency of a pedestrian to avoid dense spaces and thereby to optimize speed and travel time.

The misanthrope process is a generalisation of the zero-range and exclusion processes (Evans and Hanney; 2005; Liggett; 1985). One of its key properties is that, provided some constraints on these transition rates, it has an exact steady-state solution of factorised form that may be obtained analytically. Kanai (2010) presents a stochastic model to describe two-lane traffic on a highway, which is discretised into lane segments that can hold at most one vehicle at a time. Transition probabilities of each combination of possible states are specified to describe the propagation and lane-changing of vehicles. Tordeux et al. (2014) use a misanthrope process to model single-lane highway traffic. The model can be seen as a continuous-time extension of cellular automata, however relying on a fundamental diagram instead of a set of behavioural rules. Both analytical solutions for simple cases, as well as simulations of more general settings are discussed.

In the ensuing, a mesoscopic pedestrian flow model is presented. Pedestrians are represented explicitly as in microscopic models, and their propagation is described macroscopically by a density-speed relationship. Thus individuals can be traced throughout the system, which makes the proposed model suitable for integration within an agent-based framework, while requiring substantially less computation time than microscopic approaches. Furthermore, the model allows to capture the inherent variability of pedestrian flow, both in terms of population heterogeneity and general stochasticity. Unlike in vehicular traffic and queuing models, pedestrians are not confined to fixed lanes and can explore space freely. To illustrate the modelling approach, we rely on a minimalistic formulation, but emphasise potential generalisations wherever feasible.

\section{A mesoscopic pedestrian model}

\subsection{Definition of the model}

The proposed pedestrian model describes walking behaviour in two dimensions for a given pedestrian origindestination demand. It is defined in discrete space and continuous time. A hexagonal lattice is considered in the following, yet any lattice may be used. Unlike in the case of square grids, the definition of neighbours in hexagonal lattices is straightforward, making them a popular choice in pedestrian flow studies (Davidich and Köster; 2013; Gipps; 1986; Guo et al.; 2011; Hartmann; 2010). The length of the hexagon face is denoted by $a>0$, while the hexagon area is given by $\alpha=1.5 \sqrt{3} a^{2}$. Each hexagon can contain up to $N \geq 1$ pedestrians, depending on the pedestrian size. If we refer to the maximal density by $\rho_{\mathrm{jam}}$, then the static cell capacity is given by $N=\left\lceil\alpha \rho_{\mathrm{jam}}\right\rceil$. We denote by $n \in[0, N]$ the number of pedestrians in a given hexagon and by $n_{i}$, $i=1, \ldots 6$, the pedestrian numbers in the neighbouring cells (see Fig. 1).

As for a basic Poisson queuing process, exponential distributions describe the time between events (i.e. between pedestrian movements from one cell to another, henceforth referred to as 'jump'). However, in contrast to Poisson processes, their rate of occurrence is a dynamic quantity depending on the system state. 

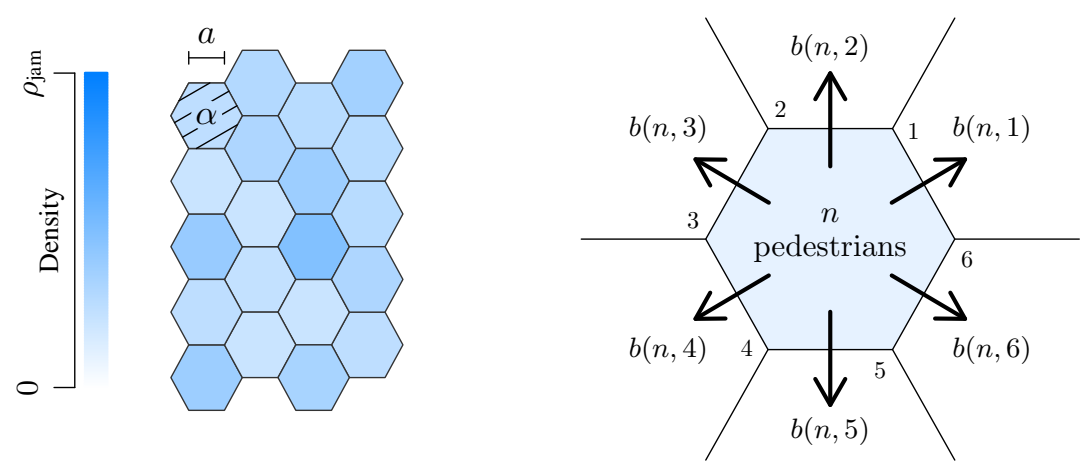

Figure 1: Space representation of the mesoscopic model. Each hexagon can contain up to $N \geq 1$ pedestrians. The integers $n$ and $\left(n_{i}\right)$ are the pedestrian numbers in a hexagon and its six neighbours. A pedestrian jumps from a hexagon to one of the neighbours according to given jump rates $b$ depending on pedestrian numbers on departure and arrival sites and on the direction.

We suppose that each pedestrian expresses an individual jump rate depending on the aggregated density level on the cells, as well as on personal characteristics. These characteristics are subsumed into classes. Each pedestrian is associated with exactly one class $\mathrm{c}$. The number of classes can be defined freely; in principle, there may be as many classes as there are pedestrians, or only a single one for the entire population. The jump rate $b^{(\mathrm{c})}$ of a pedestrian from class $\mathrm{c}$ in a hexagon with $n \in[1, N]$ pedestrians to hexagon $i$ is the product

$$
b^{(\mathrm{c})}(n, i)=\frac{\kappa}{n} J^{(\mathrm{c})}\left(n, n_{i}\right) D^{(\mathrm{c})}\left(h_{i}, J^{(\mathrm{c})}\left(n, n_{i}\right)\right),
$$

where it holds by convention $b^{(\mathrm{c})}(0, i)=0$ when no pedestrian is present (i.e. $\left.n=0\right)$. Here:

- $J^{(\mathrm{c})}\left(n, n_{i}\right)$ is the specific flow (i.e. the flow by unit of width) from the considered hexagon to hexagon $i$ in absence of any assumptions regarding walking direction,

- $D^{(\mathrm{c})}\left(h_{i}, J^{(\mathrm{c})}\right)$ is a directional factor with $h_{i}$ the direction from the considered hexagon to hexagon $i$.

The flow $J$ is supposed to be a non-decreasing function of the pedestrian number on the departure cell, and a non-increasing function of the pedestrian number on the arrival cell. It is calibrated by a given uni-modal fundamental diagram describing a phenomenological relationship between the flow and the density (Seyfried et al.; 2005; Treiber and Kesting; 2013). The factor $D$ allows to specify a desired walking direction and depends on the intended destination of the pedestrians. The parameter $\kappa=1.5 a$ is a coefficient that depends on the type of lattice. The factor 1.5 in the definition of $\kappa$ is due to hexagons being tangled. It would be 1 for a regular square lattice or $(\sqrt{2})^{-1}$ for a triangular lattice.

No first-in first out (FIFO) rules are imposed and the total jump rate from the considered cell to the cell $i$ is

$$
b(n, i)=\sum_{m=1}^{n} b^{\left(\mathbf{c}_{m}\right)}(n, i),
$$

where $m$ refers to the class number. Consequently, $b(n, i)=\kappa J^{(\mathrm{c})}\left(n, n_{i}\right) D^{(\mathrm{c})}\left(h_{i}, J^{(\mathrm{c})}\left(n, n_{i}\right)\right)$ if all the pedestrians are from the same class c (i.e. $\mathrm{c}_{m}=\mathrm{c}$ for all $m$ ). For a static cell capacity of $N=1$, a microscopic model results that represents a stochastic exclusion process (Liggett; 1985; Spitzer; 1970). This special case is briefly described below (see Sec. 4). The main part of the paper considers the case where $N$ is large, i.e., where the cell area is such that it can contain more than a single pedestrian. In this case the model is mesoscopic, and represents a stochastic misanthrope process (Cocozza-Thivent; 1985).

\subsubsection{Uni-directional case}

The specific flow $J^{(\mathrm{c})}$ for pedestrian class $c$ is the minimum between the demand $\Delta^{(\mathrm{c})}(\cdot)$ of the considered hexagon and the supply $\Sigma^{(\mathrm{c})}(\cdot)$ of the destination hexagon $i$

$$
J^{(\mathrm{c})}\left(n, n_{i}\right)=\min \left\{\Delta^{(\mathrm{c})}(n / \alpha), \Sigma^{(\mathrm{c})}\left(n_{i} / \alpha\right)\right\} .
$$

Such a flow model corresponds to the discretised macroscopic conservative equation of transport that results when using a Godunov scheme (Eymard et al.; 2012; Lebacque; 1996). The same modelling approach is used 
in Osorio et al. (2011); Tordeux et al. (2014) for homogeneous flows. The demand $\Delta^{(\mathrm{c})}(\cdot)$ and supply $\Sigma^{(\mathrm{c})}(\cdot)$ functions follow from the fundamental diagram (FD). Many shapes for the fundamental diagram exist in the literature (see, e.g., Bellomo and Dogbe (2011); Treiber and Kesting (2013)). Some approaches consider multi-directional flows (Hänseler, Lam, Bierlaire, Lederrey and Nikolić; 2017; Wong et al.; 2010; Xie and Wong; 2015) as well as heterogeneity (Corbetta et al.; 2015; Jabari et al.; 2014; Nikolić et al.; 2016). In the following, the FD is minimal and assumed triangular (Flötteröd and Lämmel; 2015; Newell; 2002), see Fig. 2, left panel. Yet any uni-modal shape may be used. The triangular FD is based on three parameters, namely the desired speed $v_{0}$ (the slope of the FD in the free state), the shock-wave speed $\gamma$ (the absolute slope in the congested state), and the maximal pedestrian density threshold $\rho_{\text {jam }}$. The demand and supply functions are respectively $\Delta(\rho)=\min \left\{Q, v_{0} \rho\right\}$ and $\Sigma(\rho)=\min \left\{Q, \gamma\left(\rho_{\text {jam }}-\rho\right)\right\}$, where $Q=v_{0} \rho_{\text {jam }} /\left(1+v_{0} / \gamma\right)$ is the maximal specific flow. Note that here the parameters $v_{0}, \gamma$ and $\rho_{\text {jam }}$ depend on the considered pedestrian class to take into account population heterogeneity. They may also depend on the location to take into account spatial dependencies due to the geometry.

The granularity of the model is determined by the hexagon lattice size $\alpha$ and the face length $a$. The granularity is maximal when the hexagon size is equal to that of a pedestrian and $N=1$ (exclusion process). Yet, special conditions for $N$ are required to well define the desired speed $v_{0}$. Specifically, the desired speed must be reachable in free-flow situations and consequently the demand $v_{0} / \alpha$ must be smaller than the maximal specific flow $Q$. This condition is $\alpha \geq v_{0} / Q$ or $N \geq 1+v_{0} / \gamma$. Such a condition does not hold for any $v_{0}, \gamma$ and $N$. In fact, the speed in the free state is effectively $v_{0}$ if the cell size (and so $N$ ) is sufficiently large. If $N<1+v_{0} / \gamma$, then the maximal speed is bounded by $v_{0} /\left(1+v_{0} / \gamma\right)$. Yet such a bound can be corrected by specifying the slope of the congested part of the fundamental diagram $\gamma$ as $\tilde{\gamma}=\max \left\{\gamma, v_{0} /(N-1)\right\}$. In such a case, the fundamental diagram is simply the linear function $\rho \mapsto v_{0} \rho$ for the exclusion process where $N=1$. It does not depend on the congested part of the FD. The speed is equal to the desired speed if the site is free and zero if it is occupied. No parameter except the exclusion rule calibrates the congested state for this special microscopic case.

The direction factor $D^{\text {(c) }}$ considers the walking direction $h$ to specify the overall movement of pedestrians. For simplicity, the direction $h$ is assumed to be exogenous, but it may also be obtained from a static floor field model, as in Burstedde et al. (2001), or from an eikonal-type equation as in Hughes (2002). Specifically, the factor for the desired direction $h$ is

$$
D^{(\mathbf{c})}\left(h_{i}, J^{(\mathrm{c})}\left(n, n_{i}\right)\right)= \begin{cases}1 & \text { if } i=\arg \max _{j} w^{(\mathrm{c})}\left(h-h_{j}\right) J^{(\mathrm{c})}\left(n, n_{j}\right), \\ 0 & \text { otherwise }\end{cases}
$$

with $w^{(\mathrm{c})}$ a weight function that may depend on the pedestrian class. In the following $w: x \mapsto 1+\cos (x)$ is taken to illustrate the model (Johansson et al.; 2007), yet other shapes may be used (for instance piecewise constant as in Helbing and Molnár (1995)). In the special case where $w(\cdot)$ is constant, pedestrians no longer have a desired direction and simply aim to maximise the flow. Such a case is illustrated in Sec. 4 (see Fig. 8, right panel).

The destination factor $D^{(\mathrm{c})}$ is zero for all neighbouring cells except the one maximising $w^{(\mathrm{c})}\left(h-h_{i}\right) J^{(\mathrm{c})}\left(n, n_{i}\right)$. If several neighbours maximise such a quantity, then only one among them is randomly chosen. Except in the latter case, the rule for the direction is strictly deterministic. Further randomness could be added by keeping small probabilities to choose one of the cells that do not maximise the weighted flow, notably to avoid deterministic gridlocks.

\subsubsection{Multi-directional case}

The multi-directional model is an extension of the uni-directional model taking into account heterogeneity in walking directions across the population, as well as additional friction effects due to multi-directional movements. The modelling approach is based on a segmentation of pedestrians by direction and, for the sake of illustration, on trapezoidal fundamental diagrams to describe the dependency of walking speed on density.

In the following, we consider pedestrian flows with $d \geq 1$ different desired directions $\left(h_{1}, h_{2}, \ldots h_{d}\right)$. The state of the system is henceforth described by the pedestrian numbers by desired direction $\left(n_{h_{1}}, \ldots n_{h_{d}}\right)$ for each hexagon. The different pedestrian numbers evolve in interaction. We denote by

$$
p_{h}=\frac{n_{h}}{\sum_{h} n_{h}}
$$

the proportion of pedestrians with desired direction $h \in\left(h_{1}, \ldots h_{d}\right)$ in the considered hexagon. The jump rate for the pedestrians with desired direction $h$ to the destination $i$ is

$$
b_{h}^{(\mathrm{c})}(n, i)=\kappa p_{h} J_{h}^{(\mathrm{c})}\left(n, n_{i}\right) D^{(\mathrm{c})}\left(h_{i}, J_{h}^{(\mathrm{c})}\left(n, n_{i}\right)\right) .
$$



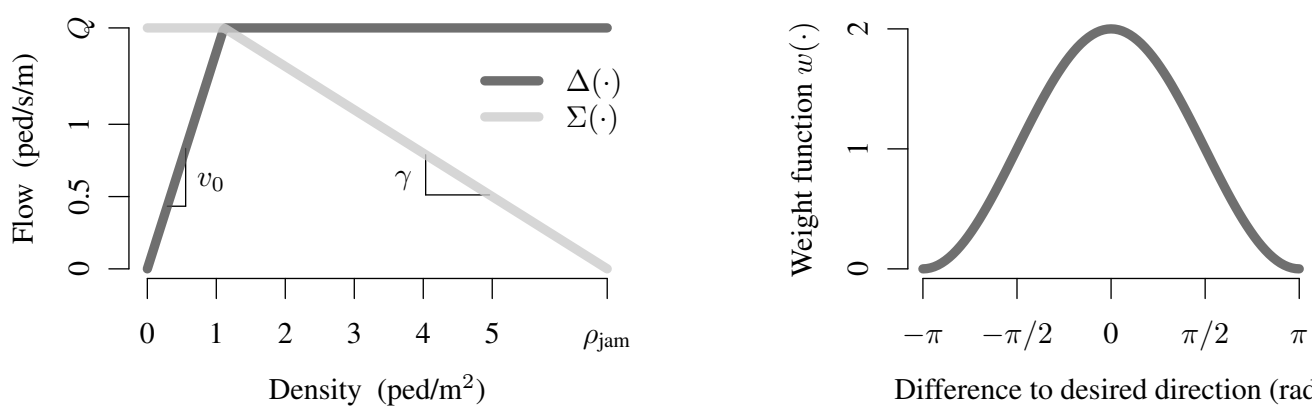

Difference to desired direction (rad)

Figure 2: Minimal example specification of model functions. Left panel: Triangular fundamental diagram with three parameters and associated demand $\Delta(\cdot)$ and supply $\Sigma(\cdot)$ functions. Right panel: Weight function $w: x \mapsto 1+\cos (x)$ for the direction factor.

In order to model friction between pedestrians with different directions, the maximal specific flow (and so the demand and supply functions), independently of the pedestrian class, is bounded by direction (Zhang et al.; 2012) (see Fig. 3)

$$
J_{h}^{(\mathrm{c})}\left(n, n_{i}\right)=\min \left\{J^{(\mathrm{c})}\left(n, n_{i}\right), \tilde{p}_{i, h} Q^{(\mathrm{c})}\right\} .
$$

Here $\tilde{p}_{i, h}=p_{0}+\left(1-p_{0}\right) p_{i, h}$ is the proportion upper to $p_{0}$, i.e. the proportion bounded to the left by $p_{0}$, of pedestrians with destination $h$ on $i, p_{i, h}$ being the corresponding actual proportion, and $p_{0} \in[0,1]$ a threshold parameter for the friction. The parameter is in the following assumed constant but in principle it may depend on the type of interactions (counter-flow, cross-flow, etc.) as well as on the pedestrian class. Trapezoidal shapes are used to describe pedestrian dynamics in Asano et al. (2007).

The multi-directional model reduces to the uni-directional one if only one desired direction $h$ exists (i.e. $d=1$ ), since in this case $p_{h}=1$ and $J_{h}=J$, see Eq. (1). For multiple directions, the jump rate decreases for a given direction $h$ when the cells are occupied by pedestrians with different directions as long as the parameter $p_{0}$ is sufficiently low. For instance, if $p_{0}=0$, the jump rates to cells that do not contain any pedestrians with the same direction are zero. Inversely, for $p_{0}=1$, pedestrians change cells with no distinction of the different pedestrian directions. In such a case, the flow $J_{h}$ becomes independent of the desired direction such that $J_{h}=J$ for all $h$. There is no more friction effect.

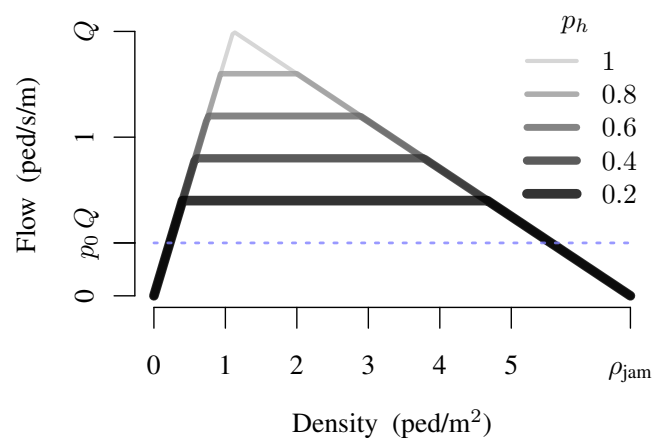

Figure 3: Fundamental diagram for a given direction $h$ according to the proportion $p^{h}$ of pedestrians with the same direction with $p_{0}=0.2$.

\subsection{Stationary distribution}

The proposed model is a Markovian process representing a finite system. Such Markovian processes can theoretically be described in stationary state by solving global balance equations that are referred to as FokkerPlanck in physics or Kolmogorov backward equations in the context of interactive particle systems. They are expressed as

$$
\frac{\mathrm{d} P(t)}{\mathrm{d} t}=P(t) Q(t),
$$


where the term $P$ describes the probability of any possible configuration, and where $Q$ represents a Markovian matrix for the jump rate from one configuration to another (given by the function $b$ ). The problem is a linear differential system, whose complexity increases exponentially with the number of possible configurations. Generally speaking, no explicit solution exists for heterogeneous systems or complex geometries. Therefore, Eq. (8) has to be approximated numerically or by simulation.

Particular processes exist for which the stationary distribution has a product form. This is notably the case for the zero-range process (see Evans and Hanney; 2005). Such a property allows to considerably simplify the solution space and to obtain explicit stationary distributions even for complex systems by using mean field theory (Schadschneider et al.; 2010). However, the misanthrope process has a product form invariant distribution if and only if the jump rate $b$ satisfies the recursive relation (Cocozza-Thivent; 1985)

$$
b(n, 0)+\frac{b(1, n)}{b(n+1,0)} \frac{b(p, 0)}{b(1, p-1)} b(n+1, p-1)=b(n, p)+b(p, 0),
$$

$n$ being the number of particles on the departure site and $p$ the number on the arrival site. The function $b$ can be constructed recursively, starting with $b(n, 0), n=1, \ldots, N$, and $b(1, p), p=0, \ldots, N-1$. The model is then characterized by the two functions $b(n, 0)$ and $b(1, p)$.

The jump rate function defined by Eq. (3) does not satisfy the above conditions. Nevertheless, one can define $b(n, 0)=\Delta(n / D)$ and $b(1, N)=\Sigma(p / N)$ based on the triangular fundamental diagram and compute the other values $b(n, p)$ according to Eq. (9). Then, the uni-dimensional stochastic process admits a product stationary measure. However, the validity of this reasoning is questionable. Fig. 4 presents the jump rate function for the triangular fundamental diagram, Eq. (3), and for the associated measure product recursive condition, Eq. (9). The form induced by a product stationary measure is not reasonable since the jump rate is approximately nil as soon as the density is higher than the critical density threshold.

As such, Eq. (9) is restrictive and implies a non-linear shape of the jump rate. Consequently, only basic systems can be analysed theoretically (e.g. homogeneous systems on a single site, as in Kanai; 2010; Osorio et al.; 2011; Tordeux et al.; 2014). In contrast, the experiments we carry out in the following are relatively complex, involving multi-directional flow and non-trivial geometries. They can in general not be solved explicitly, and instead have to be investigated numerically by simulation. A simulation-based formulation of the model is described below.


Figure 4: Triangular fundamental diagram, Eq. (3), and corresponding fundamental diagram for the measure product recursive condition, Eq. (9). Left panel: Flow/density plot. Right panel: Speed/density plot. The form induced by the product stationary distribution is not reasonable since the jump rate is approximately nil as soon as the density is higher than the critical density threshold.

\subsection{Simulation-based formulation}

The simulation of the model is straightforward. It can be carried out in continuous time, without any requirement of discretisation. Each hexagon containing at least one pedestrian has a continuous exponential time $T_{0}$ determining the next jump time. The jump time $T_{0}$ depends on the system state through the jump rate $b$

$$
T_{0}=t+\mathcal{E}(b),
$$

with $\mathcal{E}(b)$ representing an independent exponential random variable with mean $1 / b$ and $t$ denoting the global simulation time. The simulation of the process is event-based. It involves the iterative application of the three operations (see Fig. 5) 
1. Select the cell with minimal time;

2. Execute the jump and update the time;

3. Calculate the new jump times of the cells where the jump rate changed.

At each jump, only the jump times of the cells where the jump rate changed have to be updated. Therefore, the computational effort is proportional to the flow and not directly to the density. This can make the approach faster than classical models, especially at high density levels.

Note that the model could be defined in discrete time as well. In this case, if we denote the time step by $\delta t$, the jump times of the pedestrians are geometrically distributed with parameter $p=b \delta t$. The time step needs to be chosen such that the Courant-Friedrichs-Lewy (CFL) condition holds, i.e., $p=b \delta t \leq 1$ (Courant et al.; 1928).

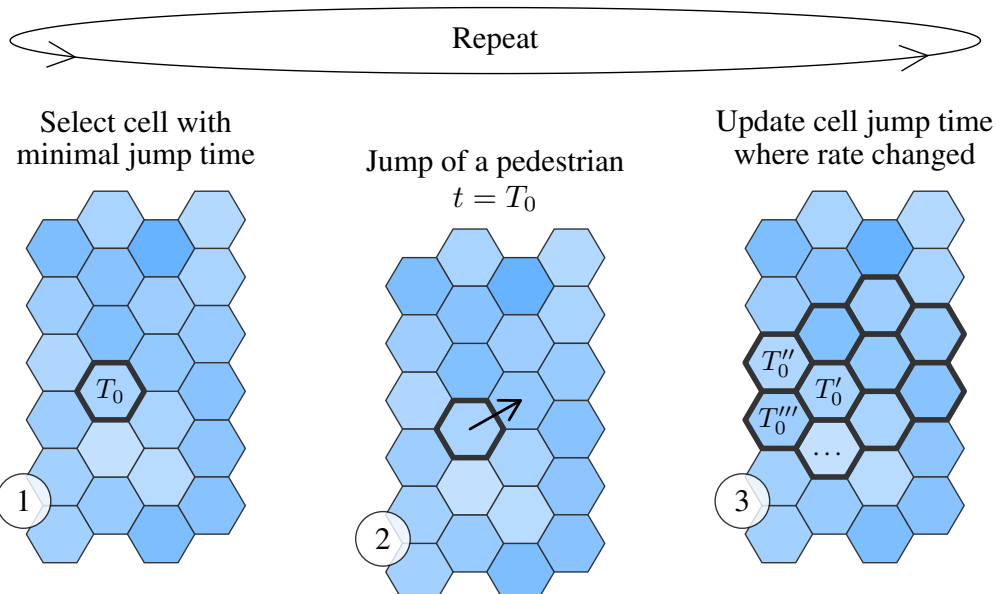

Figure 5: Scheme for the simulation of the model. Each hexagon with at least one pedestrian has initially a continuous jump time $T_{0}>0$. The simulation consists of repeating the three steps: 1 . Select cell with minimal jump time; 2 . Do the jump and set the global time to the selected cell's jump time; 3. Update the jump times of the cells where the rate changed.

\section{Numerical experiments}

In the following, rectangular walkways with a width and a length of approximately 25 and $50 \mathrm{~m}$ and periodic up/down and right/left boundary conditions are simulated from random initial conditions. Different scenarios of uni-directional and counter-flow with or without obstacles are tested. A single pedestrian class is considered with parameters $v_{0}=1.5 \mathrm{~m} / \mathrm{s}, \gamma=0.3 \mathrm{~m} / \mathrm{s}$ and $\rho_{\mathrm{jam}}=6.667 \mathrm{ped} / \mathrm{m}^{2}$ (see Fig. 2, left panel). The system may contain up to 8000 pedestrians at jam density. Three global variables are used to describe the performance of the model.

1. The mean density $\bar{\rho} \in\left[0, \rho_{\text {jam }}\right]$ is defined by

$$
\bar{\rho}=\frac{1}{K} \sum_{k=1}^{K} n_{k} / \alpha_{k},
$$

$\alpha_{k}$ being the area and $n_{k}$ the pedestrian number of hexagon $k . K$ is the number of hexagons in the system.

2. The empirical standard deviation $\bar{\sigma} \geq 0$ of the density is such that

$$
\bar{\sigma}^{2}=\frac{1}{K} \sum_{k=1}^{K}\left(n_{k} / \alpha-\bar{\rho}\right)^{2} .
$$


3. The mean absolute specific flow $\bar{J} \in[0, Q]$ (i.e. the flow in any direction) is given by

$$
\bar{J}=\frac{1}{K \kappa} \sum_{k=1}^{K} \sum_{i=1}^{6} b\left(n_{k}, i_{k}\right)
$$

$\left(i_{k}\right)$ being the six neighbouring hexagons of hexagon $k$. Note that the temporal mean flow $\bar{J}_{t}=\frac{1}{t \tau} S_{t}$, $S_{t}$ being the number of pedestrians leaving a given hexagon during time interval $[0, t]$, tends to the asymptotic spatial mean flow $\bar{J}$ as $t \rightarrow \infty$ (Cocozza-Thivent; 1997).

\subsection{Fundamental diagram}

The pedestrian dynamics are aggregated when the cell size is large compared to the pedestrian size, i.e. for $N \gg 1$. In this case, it is well known that the model dynamics in one dimension tend to the solution of the classical deterministic continuity equation of transport as the cell size increases (Eymard et al.; 2012; Tordeux et al.; 2014). The results obtained with the bi-dimensional pedestrian model are similar. Snapshots of the systems in stationary state (i.e. for simulation time higher than 500,000 s) for $a=1,2$, and $4 \mathrm{~m}$ (corresponding to $N=17,69$ and 277, respectively) are presented in Fig. 6 . The pedestrian distribution tends to be uniform as the hexagon size increases. The specific flow and density standard deviation in stationary state are plotted as a function of the density in Fig. 7. The flow tends to the deterministic fundamental diagram parameter and the density standard deviation tends to zero as $\alpha \rightarrow \infty$. Therefore, the variability in the system can be controlled by the cell size.
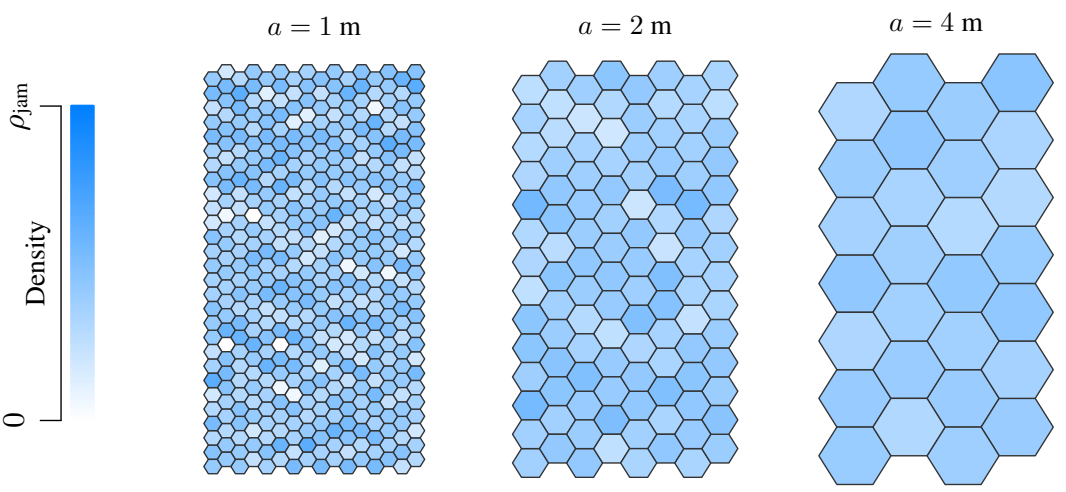

Figure 6: Snapshots of uni-directional systems in stationary state for different hexagon sizes for $\rho=2.5 \mathrm{ped} / \mathrm{m}^{2}$ (i.e. $\approx 3000$ ped). The density across hexagons tends to be homogeneous as the hexagon size increases.
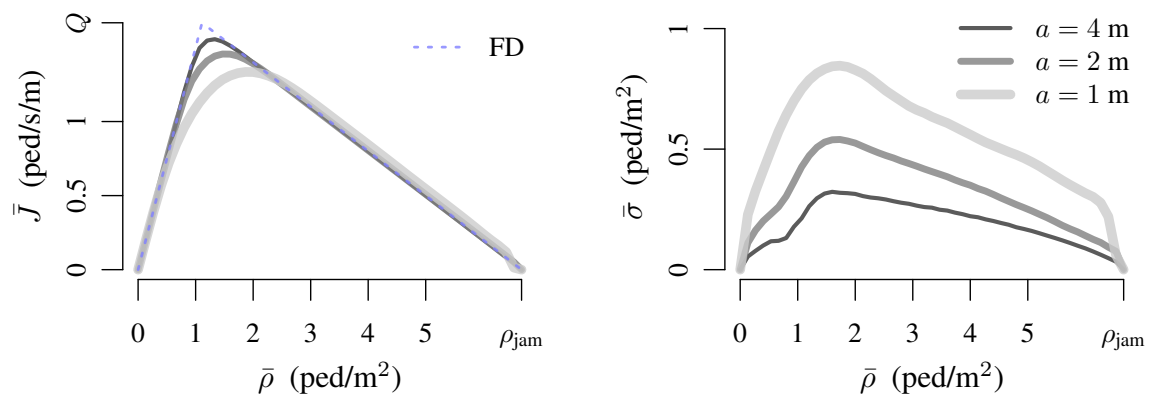

Figure 7: Mean specific flow and density standard deviation as a function of the density in the uni-directional systems in stationary state for different hexagon sizes. The density-flow relationship tends to the deterministic fundamental diagram and the pedestrian repartition tends to be homogeneous as the hexagon size increases. 


\subsection{Congestion and rarefaction due to obstacles}

An obstacle can be included in a cell by limiting the supply and the demand, or it can replace a cell altogether by setting these parameters to zero. In the latter case, there is no pedestrian in an obstacle cell and no pedestrian can jump into it. Depending on the shape of the weight function for the desired direction $w(\cdot)$, congestion can appear upstream to obstacles, and rarefaction can occur downstream (see Fig. 8, left panels). If the direction weight $w(\cdot)$ is a constant function, the distribution of the density is uniform even in presence of obstacles, and the mean specific flow is the one of a system with no obstacle (see Fig. 8, right panels).

From North to South
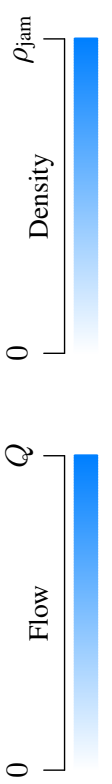
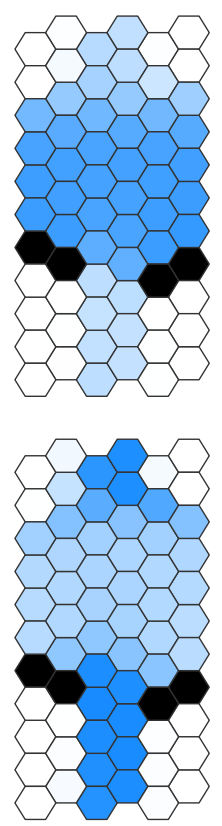
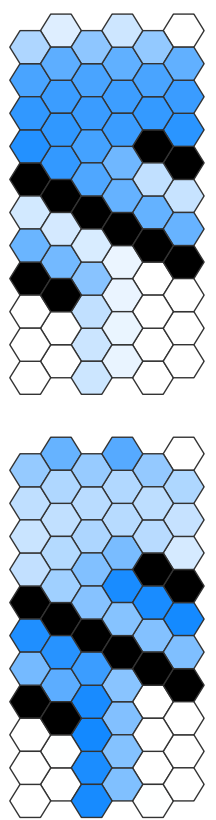

No desired direction
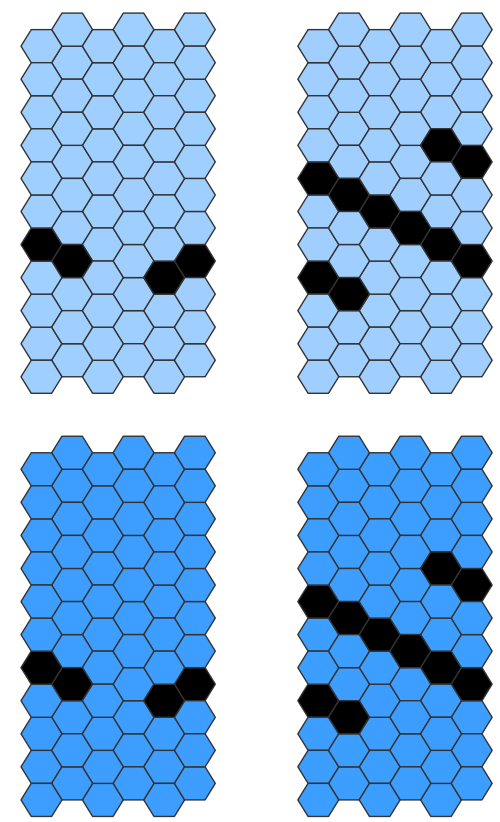

Figure 8: Mean density (top panels) and specific flow (bottom panels) for a uni-directional system with obstacles in stationary state for $\rho=2.5 \mathrm{ped} / \mathrm{m}^{2}$ and $a=2.5 \mathrm{~m}$. The boundaries are periodic. The obstacles generate congestion and rarefaction when the desired direction is modelled (i.e. for $w(x)=1+\cos (x)$, left panels), while the state is uniform if the weight $w$ is constant (right panels).

\subsection{Counter-flow and lane formation}

Counter-flows are considered in the following experiments. Half of the pedestrians have the desired direction $h_{1}=-\pi / 2$, the others having the opposite desired direction $h_{2}=\pi / 2$. The initial configuration is random. Three experiments are carried out. In the first one, we consider the limit case $p_{0}=1$, while the second and third experiments consider friction effects with $p_{0}=0.5$ and $p_{0}=0.2$. No lanes emerge and a gridlock appears for $p_{0}=1$, see Fig. 9. Self-organisation of the system in rectilinear lanes by direction is observed for $p_{0}=0.5$ and $p_{0}=0.2$ in stationary state (see respectively Figs. 10 and 11). The flow for this configuration is close to the uni-directional FD. However, the time required to get lanes can be important when the friction is too high (i.e. for too low values of $p_{0}$ ). The case $p_{0} \approx 0$ (as the case $p_{0} \approx 1$ with no friction) can even lead to gridlocks. Therefore only specific bounds of the fundamental diagram allow for lane formation.

\subsection{Sensitivity analysis}

The specific flow and density standard deviation for the bi-directional system in stationary state are plotted in Fig. 12 as a function of the friction parameter $p_{0}$, the hexagon face length $a$, and the density $\rho$ (with default values $p_{0}=0.2, a=2.5 \mathrm{~m}$ and $\rho=2.5 \mathrm{ped} / \mathrm{m}^{2}$ ). 50 experiments are realised for each parameter value. Flow and density standard deviation are close to the values observed for a uni-directional system when the lanes by direction are present.

All the three parameters $p_{0}, a$, and $\rho$ describe a transition for the occurrence of lanes. As already shown in Figs. 9 and 11, the threshold $p_{0}$ has to be sufficiently small for lanes to appear (approximately $p_{0}<0.6$ in 

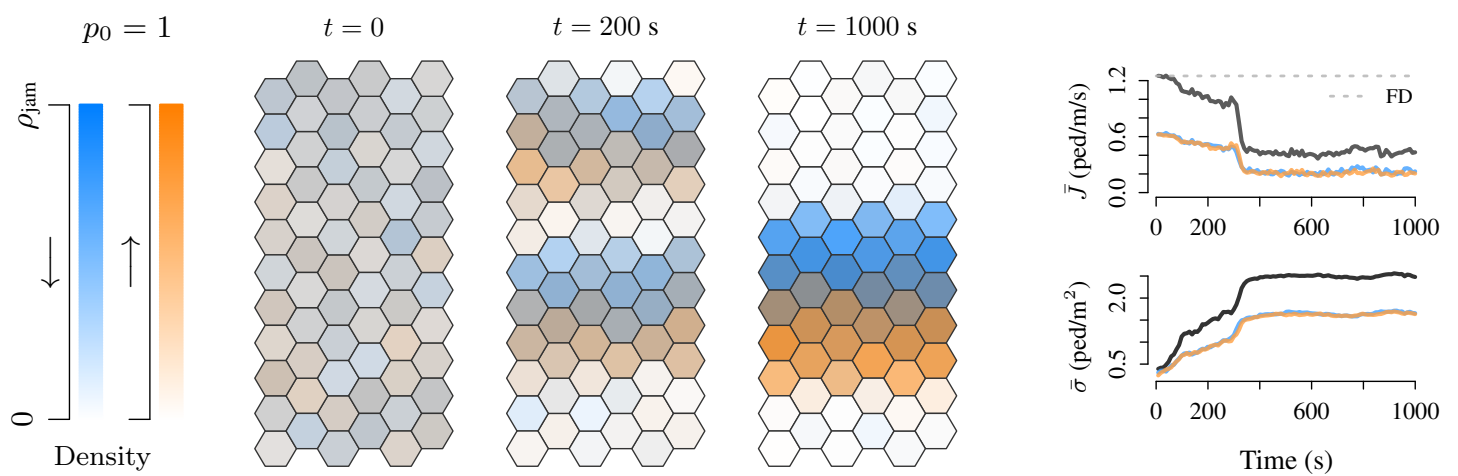

Figure 9: Density snapshots and specific flow and density standard deviation sequences for a bi-directional system with $p_{0}=1, \rho=2.5 \mathrm{ped} / \mathrm{m}^{2}$, and $a=2.5 \mathrm{~m}$. Up, down, right and left boundaries are periodic. The absence of friction between pedestrians in opposing directions does not allow for the emergence of lanes by direction and a gridlock appears.
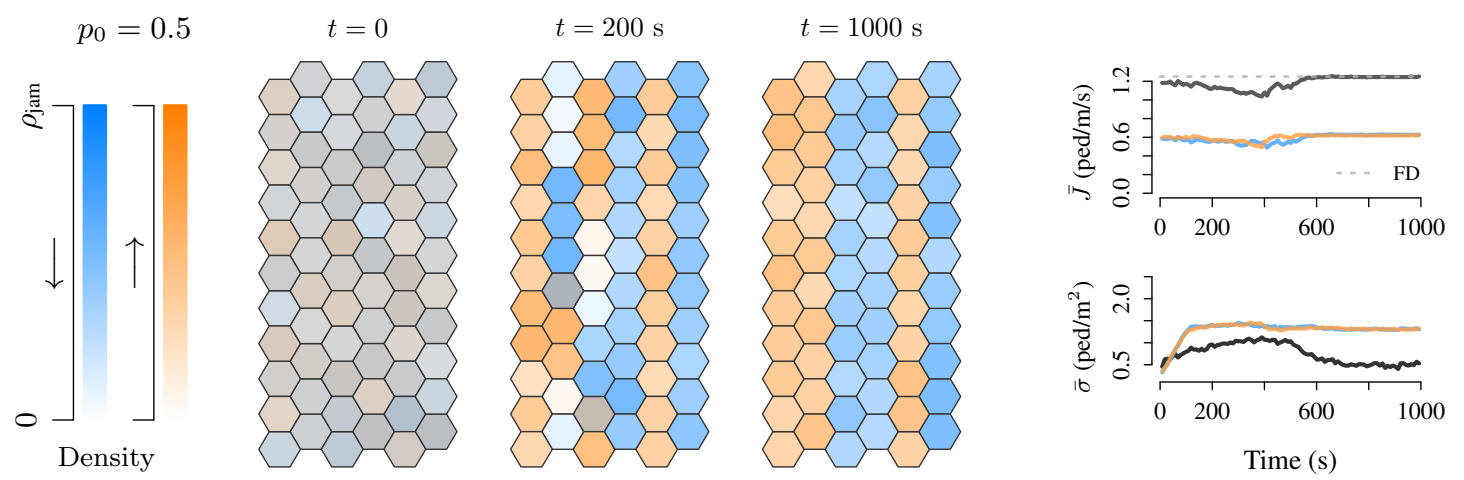

Figure 10: Density snapshots and specific flow and density standard deviation sequences for a bi-directional system with $p_{0}=0.5, \rho=2.5 \mathrm{ped} / \mathrm{m}^{2}$, and $a=2.5 \mathrm{~m}$. Up, down, right and left boundaries are periodic. The explicit consideration of friction between pedestrians in opposing directions in the supply functions allows for the emergence of lanes by direction.
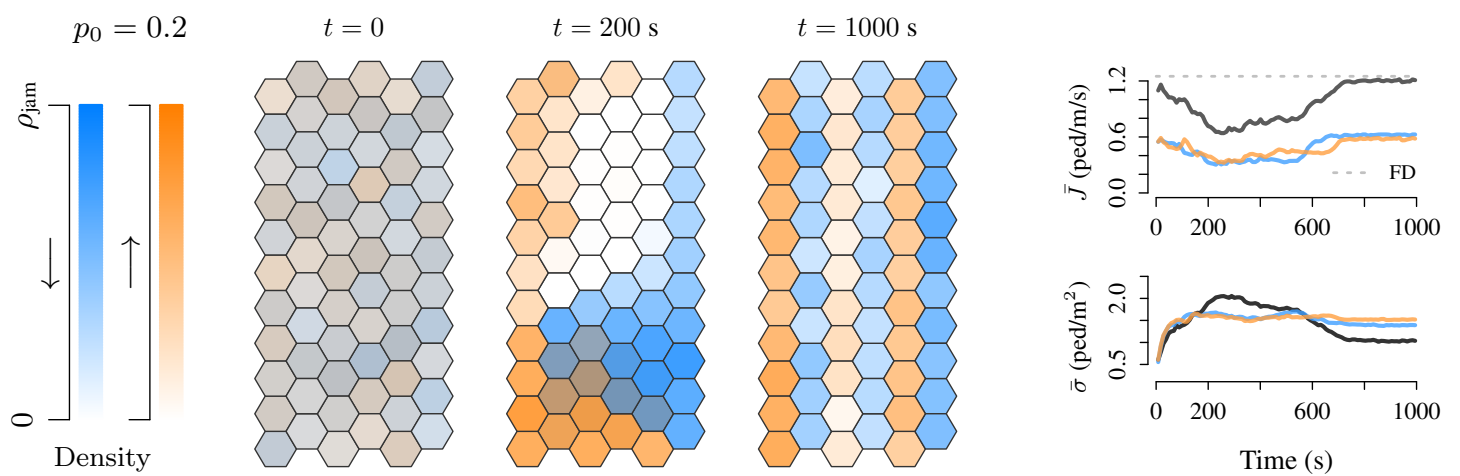

Figure 11: Density snapshots and specific flow and density standard deviation sequences for a bi-directional system with $p_{0}=0.2, \rho=2.5 \mathrm{ped} / \mathrm{m}^{2}$, and $a=2.5 \mathrm{~m}$. Up, down, right and left boundaries are periodic. Too high frictions (i.e., too low values of $p_{0}$ ) limit the lane formation and can lead to gridlock.

this example, see Fig. 12, left panels). More precisely, the system still yields gridlocks and the flow is low if the friction is too high, i.e. for $p_{0} \approx 0$, while the flow is maximised for approximately $p_{0}=0.5$.

The lane formation phenomenon also depends on the cell size. As shown in Fig. 12, middle panels, lane formation appears if the hexagon size is sufficiently high. For $a=1 \mathrm{~m}$ the simulations always yield a gridlock while lanes systematically emerge for approximately $a \geq 2 \mathrm{~m}$. As shown in Figs. 6 and 7, the variability of the density in the model is controlled by the cell size. Therefore the lane formation appears if 
the randomness is sufficiently low. This phenomenon is similar to the 'freezing by heating' effect observed within microscopic force-based models with additive noise (Helbing et al.; 2000b).

The density impacts the lane formation (see Fig. 12, right panels). Three states can be identified. For very low densities (approximately $\rho<0.5 \mathrm{ped} / \mathrm{m}^{2}$ in this example) both uni and bi-directional systems systematically describe similar performances. For intermediate densities (here $0.5<\rho<4 \mathrm{ped} / \mathrm{m}^{2}$ ), lane formation occurs but the system may also yield partial gridlocks. Certain density levels (around $\rho=1.8 \mathrm{ped} / \mathrm{m}^{2}$ ) almost always describe the same configuration where $1 / 3$ of the width of the system is gridlocked. Lane formation disappears for very large density levels (approximately $\rho=4 \mathrm{ped} / \mathrm{m}^{2}$ in this example, it is $\rho=6 \mathrm{ped} / \mathrm{m}^{2}$ for $a=4 \mathrm{~m}$ ), which is sometimes referred to as break-down of self-organisation. Note that further experiments not presented here have been carried out with bigger systems. Similar results have been obtained.
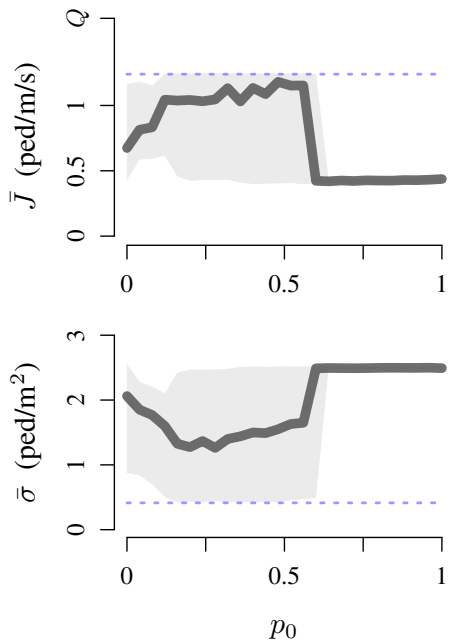
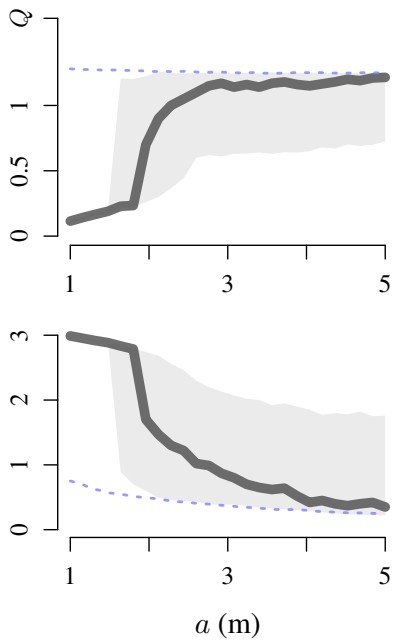
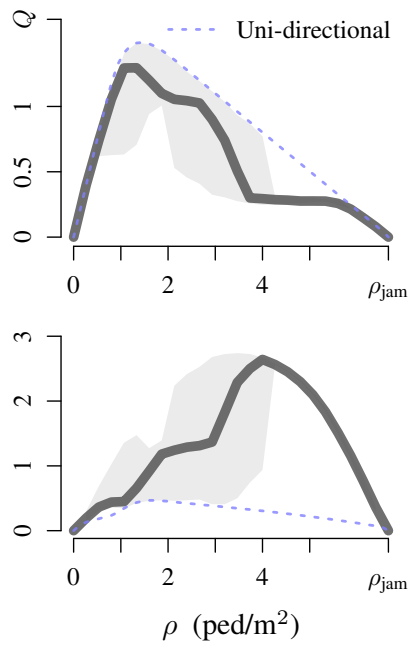

Figure 12: Specific flow (top panels) and density standard deviation (bottom panels) for bi-directional systems in stationary state as a function of $p_{0}$ (left panels), $a$ (middle panels), and $\rho$ (right panels) (default values $p_{0}=0.2, a=2.5$ $\left.\mathrm{m}, \rho=2.5 \mathrm{ped} / \mathrm{m}^{2}\right) .50$ experiments have been realised for each parameter value. Continuous lines: Mean value; Grey areas: Min/max range. Flow and density standard deviations are close to those of a uni-directional system when the lanes by direction are present.

\section{Calibration and validation of the model}

We calibrate and compare the proposed model with real data obtained under laboratory conditions. We first estimate model parameters with observations from experiments of uni-directional flows in a closed geometry, before evaluating the model with bottleneck, counter- and cross-flow experiments. A cross-validation on independent data is then made. Travel and egress times are considered for the validation. The data stems mainly from the Hermes collaborative project (2008-2011). Experiments with closed and open geometries, bottlenecks and counter-flow have been carried out with up to 400 participants, and trajectories have been collected from video recordings (Boltes et al.; 2010). A cross-flow experiment conducted by Plaue et al. (2012) is considered in Section 5.4, where also a comparison with a microscopic pedestrian flow model is presented.

\subsection{Estimation of model parameters}

The mesoscopic model for uni-directional flow solely depends on the parameters related to the fundamental diagram. In the following, a single class of parameter values is estimated. The estimation of parameters for multiple pedestrian classes is still an open research topic that exceeds the scope of this work. The parameters of the single-class specification are namely the desired speed $v_{0}$, the shock-wave speed $\gamma$, and the jam density $\rho_{\mathrm{jam}}$, grouped in the vector $\mathbf{p}=\left(v_{0}, \gamma, \rho_{\mathrm{jam}}\right)$. The experiments used for the model calibration were carried out in a closed oval geometry with density levels varying from approximately 0.25 to $4 \mathrm{ped} / \mathrm{m}^{2}$ (see Fig. 13). Two sets of 7 and 8 experiments were performed on geometries with widths $\omega_{1}=1.4 \mathrm{~m}$ and $\omega_{2}=1.8 \mathrm{~m}$ with respectively $N_{1}=10,20,45,65,85,110,130$ and $N_{2}=15,30,60,85,110,140,170,230$ participants. Pseudo-independent measurements of the specific flow and the density based on the Voronoi smoothing 
method (Steffen and Seyfried; 2010) are extracted from the trajectories by taking one measurement every 2 seconds (other sampling intervals varying from 1 to 10 seconds have been tested with similar results). The measurement is done in the centre of the geometry to avoid boundary effects with the walls. Altogether, $K=719$ observations for the pair $\left(J_{k}, \rho_{k}\right)$ of specific flow and density are obtained (see Fig. 14, left panel).

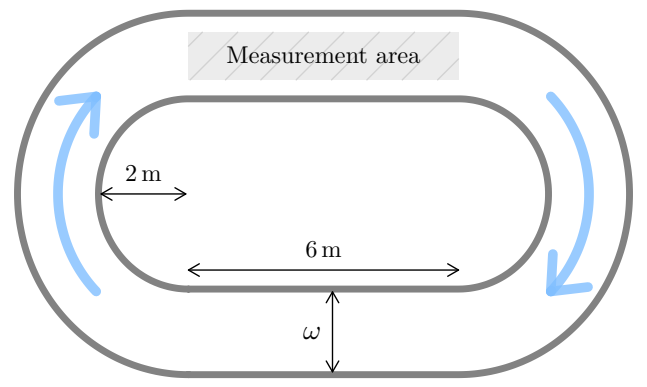

Figure 13: Space configuration of the experiment with uni-directional flows in an oval.

The parameters $\mathbf{p}=\left(v_{0}, \gamma, \rho_{\mathrm{jam}}\right)$ are estimated using the Gauss-Newton non-linear least squares method. Such an algorithm is based on the first derivative of the function to optimise. Yet, the derivatives are not defined anywhere with a strictly triangular relation. Therefore, we use the smooth triangular relationship

$$
J_{\mathbf{p}}^{\epsilon}(\rho)=\rho \epsilon \gamma \rho_{\mathrm{jam}} \ln \left(1+\exp \left(-\ln \left(1+\exp \left(-\frac{1}{\epsilon}\left[\frac{1}{\rho}-\frac{1+\frac{v_{0}}{\gamma}}{\rho_{\mathrm{jam}}}\right]\right)\right)+\frac{v_{0}}{\epsilon \gamma \rho_{\mathrm{jam}}}\right)\right) .
$$

Such a relation is a smoothed version of the triangular relation $J_{\mathbf{p}}(\rho)=\min \left\{\rho v_{0}, \max \left\{0, \gamma\left(\rho_{\text {jam }}-\rho\right)\right\}\right\}$ in the sense that $J_{\mathbf{p}}^{\epsilon}(\rho) \rightarrow J_{\mathbf{p}}(\rho)$ as $\epsilon \rightarrow 0$ for any $\rho$ and $\mathbf{p}$. We set $\epsilon=0.005 \mathrm{~m}^{2}$ in the following.

The estimation of the parameters is a local approximation of the problem

$$
\hat{\mathbf{p}}=\arg \min _{\mathbf{p}} \sum_{k=1}^{K}\left(J_{\mathbf{p}}^{\epsilon}\left(\rho_{k}\right)-J_{k}\right)^{2}
$$

The estimates with 0.95 asymptotic confidence intervals are provided in Tab. 1. Various initial parameter values have been tested with similar estimates, attesting that a global minimum is reached. The estimations are consistent with the ones obtained by Weidmann (1992) $\left(\hat{v}_{0}^{\prime}=1.34 \mathrm{~m} / \mathrm{s}, \hat{\rho}_{\text {jam }}^{\prime}=5.4 \mathrm{ped} / \mathrm{m}^{2}\right.$ and, after linearisation, $\left.\hat{\gamma}^{\prime}=0.47 \mathrm{~m} / \mathrm{s}\right)$, or the ones presented by Hänseler, Lam, Bierlaire, Lederrey and Nikolić (2017). The histogram of the residuals $J_{\mathbf{p}}^{\epsilon}\left(\rho_{k}\right)-J_{k}$ is plotted in Fig. 14, right panel. A compact distribution with no outliers is visible, corroborating the quality of the estimation.
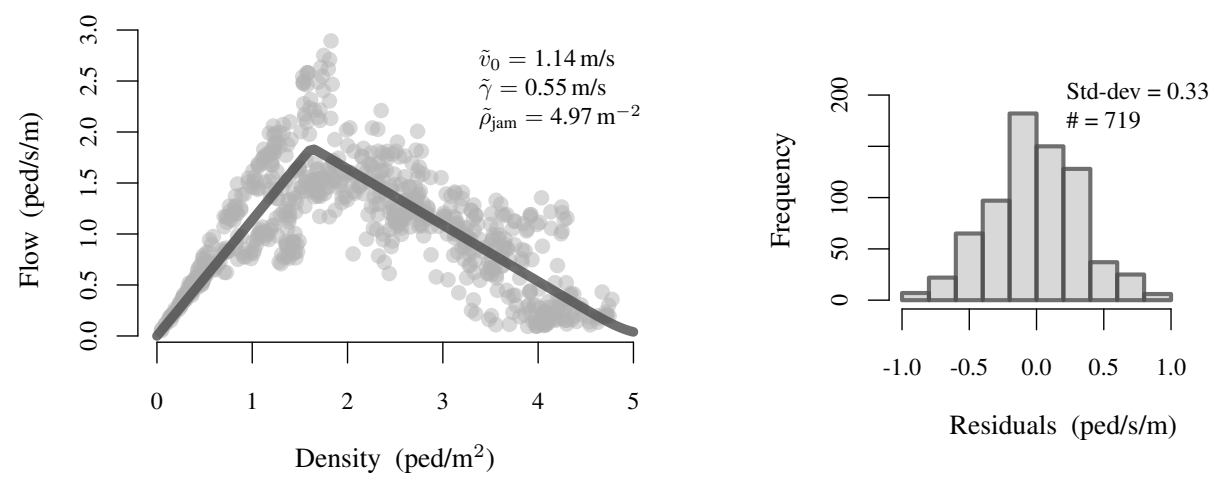

Figure 14: Flow/density measurements and the triangular fitting (left panel). Histogram of the residuals (right panel). The R-squared coefficient for the estimation is $R^{2}=0.66$ while all the Fisher tests for the parameters are significant.

The flow/density observations and simulation results of the calibrated model with $a=2 \mathrm{~m}$ are presented in Fig. 15. Similar global trends are observed as can be seen by the curves representing mean values by class of density, ranging from 0 to 5 by steps of $0.5 \mathrm{ped} / \mathrm{m}^{2}$. This is not surprising since the model is calibrated on this sample of data. It is, however, interesting to observe that the dispersion of the data is relatively well 


\begin{tabular}{l|c|c|c} 
Estimation Parameter & $v_{0}(\mathrm{~m} / \mathrm{s})$ & $\gamma(\mathrm{m} / \mathrm{s})$ & $\rho_{\text {jam }}(\mathrm{ped} / \mathrm{m})$ \\
\hline $\begin{array}{l}\text { Least squares estimate } \\
\text { 0.95 confidence interval }\end{array}$ & $\begin{array}{c}1.14 \\
{[1.08 ; 1.19]}\end{array}$ & $\begin{array}{c}0.55 \\
{[0.50 ; 0.61]}\end{array}$ & $\begin{array}{c}4.97 \\
{[4.79 ; 5.17]}\end{array}$
\end{tabular}

Table 1: Parameter estimation by non-linear least squares with 0.95 asymptotic confidence intervals.

reproduced as well (see the coloured areas describing the standard deviations around the mean values). Note that however, as shown above in Sec. 4, the variability depends on the size of the hexagon.

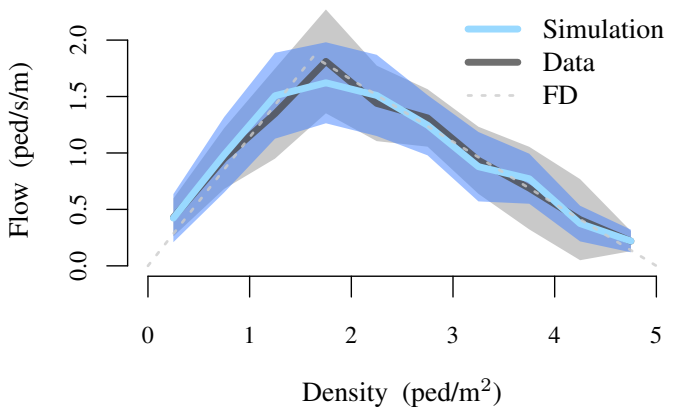

Figure 15: Flow/density observations and simulation results of the calibrated model with $a=2 \mathrm{~m}$. The curves and the coloured area represent respectively the mean value and the standard deviation by class of density (ranging from 0 to 5 by steps of $0.5 \mathrm{ped} / \mathrm{m}^{2}$ ).

\subsection{Bottleneck experiment}

The calibrated model is tested on bottleneck experiments. The geometry here is open: Pedestrians start from a waiting area, cross a corridor that ends in a bottleneck, before leaving the experiment (see Fig. 16). The corridor's width amounts to $1.8 \mathrm{~m}$ and its length to $8 \mathrm{~m}$. Three experiments are available with bottleneck widths of $\omega_{1}=0.95 \mathrm{~m}, \omega_{2}=1.20 \mathrm{~m}$ and $\omega_{3}=180 \mathrm{~m}$ with respectively $N_{1}=159, N_{2}=170$ and $N_{3}=220$ participants initially disposed in the waiting area. Trajectories are recorded within the corridor (see striped grey area in Fig. 16).

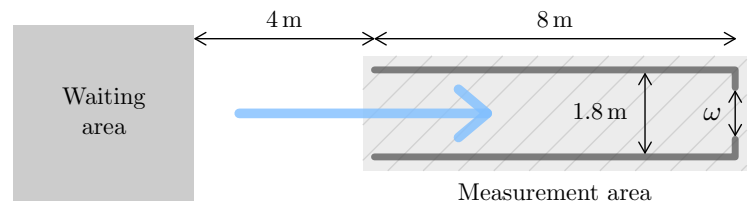

Figure 16: Space configuration of the experiment with uni-directional flows in a bottleneck.

The bottleneck configuration is implemented by a single row of four aligned hexagons. The face of the hexagons is $a=1.2 \mathrm{~m}$. The width of the modelled corridor is the one of the experiment $(1.8 \mathrm{~m})$ while its length is slightly larger $(8.31 \mathrm{~m}$ instead of $8 \mathrm{~m})$. The bottleneck is modelled by bounding the jump rate for the last hexagon according to the bottleneck width as described in Sec. 4. Monte Carlo simulations are carried out with the same initial configuration as in the real experiment. The distribution of observed and simulated travel times are relatively close (Fig. 17, top panels). Student and Fisher tests are carried out for the mean and variance obtained in the data and by simulation (see Table. 2). Except for the variance of the experiment with bottleneck width $\omega=1.8 \mathrm{~m}$, the tests are not significant. Therefore, with the exception of the latter case, one cannot reject the assumption that the mean and variance are statistically the same in the data and in the simulation.

The mean simulated egress times are relatively close to the observed ones (approximately $99 \mathrm{~s}$ on average for the simulation and $90 \mathrm{~s}$ for the experiment for $\omega_{1}=0.95 \mathrm{~m}, 86$ and $82 \mathrm{~s}$ for $\omega_{2}=1.2 \mathrm{~m}$ and 98 and $92 \mathrm{~s}$ for $\omega_{3}=1.8 \mathrm{~m}$ ). Since the model is stochastic, the egress times vary in the simulation from one run 

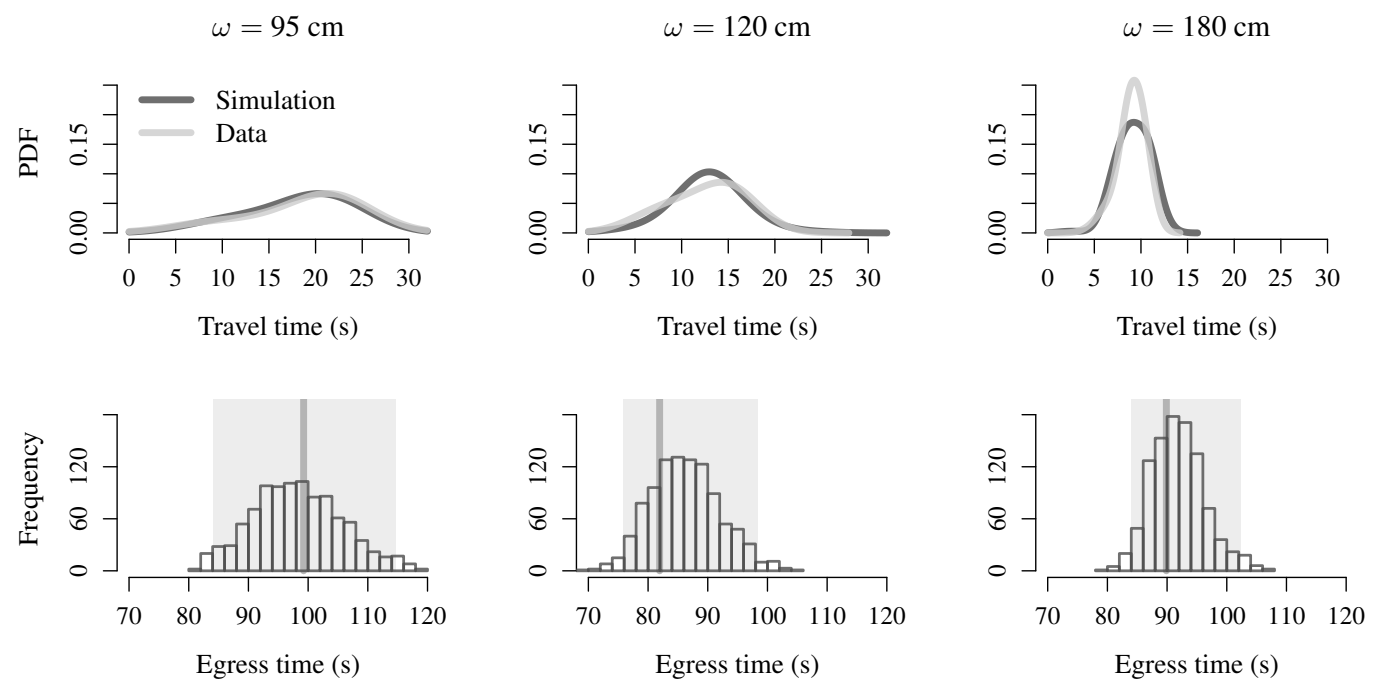

Figure 17: PDF of the travel times (top panels) and histograms of the simulated egress times (bottom panel) for the different bottleneck widths. Top panels: The dark grey curves correspond to the simulation while the light grey curves correspond to the data. Bottom panel: The grey vertical lines are the observed times while the light grey areas correspond to the 0.95 confidence intervals of the simulations.

\begin{tabular}{c|cc|cc|cc} 
& \multicolumn{2}{|c|}{$\omega=0.95 \mathrm{~m}$} & \multicolumn{2}{c|}{$\omega=1.2 \mathrm{~m}$} & \multicolumn{2}{c}{$\omega=1.8 \mathrm{~m}$} \\
\cline { 2 - 7 } & Data & Simulation & Data & Simulation & Data & Simulation \\
\hline Mean & 18.18 & 18.60 & 12.91 & 12.52 & 9.18 & 9.00 \\
Std. dev. & 1.49 & 1.53 & 1.75 & 1.88 & 1.73 & 1.34 \\
\hline Equality-test & Mean & Std. dev. & Mean & Std. dev. & Mean & Std. dev. \\
P-value & 0.47 & 0.18 & 0.27 & 0.06 & 0.23 & 0.00
\end{tabular}

Table 2: Bottleneck experiment. Mean, standard deviation (in seconds) and p-value of the equality-tests for the travel time in the data and for the simulation. The numbers of observations are respectively $N=159,170$ and 220 .

to another. It is likely also the case in the real experiment. The histograms of the simulated egress times for 1000 runs are presented in Fig. 17, bottom panels, for the three different bottleneck widths. The observed egress time (the dark grey vertical line) is compared to the 0.95 confidence intervals of the simulations (the light grey areas). The simulated egress time varies by approximately $20 \%$ and the observed times always fall within the confidence intervals.

\subsection{Counter-flow experiment}

Counter-flow experiments are investigated to examine the performance of the model in case of multi-directional movements. A corridor geometry with two opposite waiting areas is considered. The participants, at the beginning in one of the lateral waiting areas, have to cross the corridor and leave the experiment on the opposite side (see Fig. 18). The corridor width is $3.6 \mathrm{~m}$ and its length $8 \mathrm{~m}$. Several experiments with different entrance widths $\omega$ and different participant numbers are available. We consider here the experiment with an entrance width $\omega=1.2 \mathrm{~m}$ and 300 participants (i.e., 150 on each side). Trajectories at the corridor inlet as well as within the corridor have been recorded (see the striped grey area in Fig. 18).

To model the counter-flow, the same hexagon size and model parametrisation as for the previous experiment are considered. Simulations are carried out for friction parameter $p_{0}=0.2,0.5$ and 1 . As observed in the preliminary simulation results presented in Sec. 4 (see Figs. 9 and 11), lanes per direction emerge for $p_{0}=0.2$. Yet this parametrisation also yields temporary gridlocks with large travel and egress times (see Fig. 19, left panels). More stable realistic behaviours are obtained for $p_{0}=0.5$ (see Fig. 19, middle panels), while the travel and egress times are underestimated and no lanes by direction emerge in the total absence of friction in the cell supply (i.e. for $p_{0}=1$ ), see Fig. 19, right panels. Student and Fisher tests for the mean and variance of the travel times in the data and for the simulation are presented in Table 3 . The results confirm that setting the friction to $p_{0}=0.5$ yields good agreement with the data. 


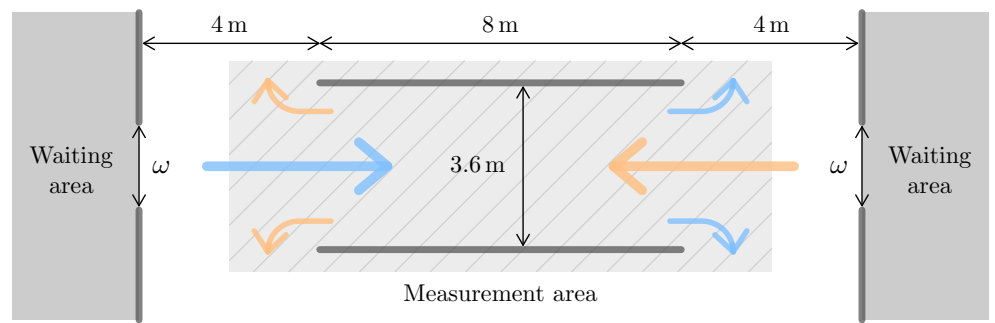

Figure 18: Space configuration of the experiment with counter-flows in a corridor.
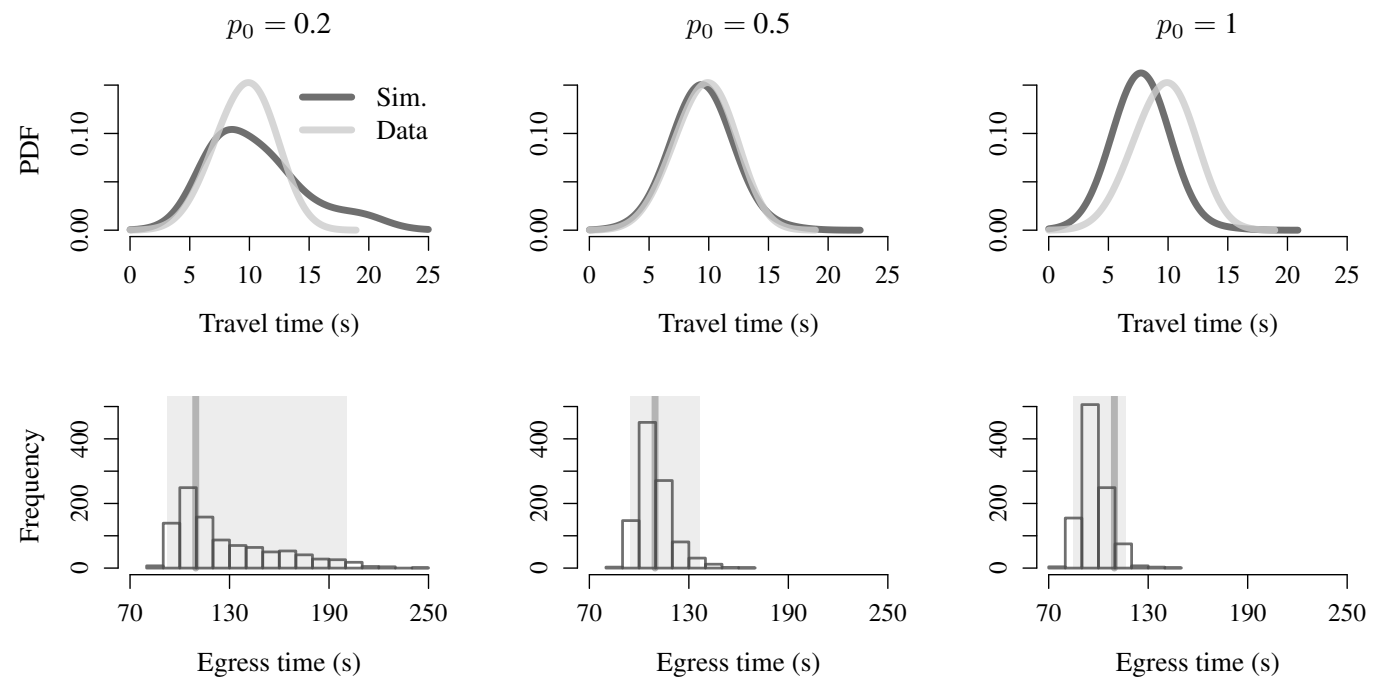

Figure 19: PDF of the travel times (top panels) and histograms of the simulated egress times (bottom panel) for $p_{0}=0.2$, $p_{0}=0.5$ and $p_{0}=1$. Top panels: The dark grey curves correspond to the simulation while the light grey curves correspond to the data. Bottom panel: The grey vertical lines are the observed times while the light grey areas correspond to the 0.95 confidence intervals of the simulations.

\begin{tabular}{c|cc|cc|cc} 
& \multicolumn{2}{|c|}{$p_{0}=0.2$} & \multicolumn{2}{|c|}{$p_{0}=0.5$} & $p_{0}=1$ & \\
\cline { 2 - 7 } & Data & Simulation & Data & Simulation & Data & Simulation \\
\hline Mean & 10.66 & 9.65 & 9.48 & 9.65 & 7.76 & 9.65 \\
Std. dev. & 1.43 & 1.13 & 1.37 & 1.27 & 1.54 & 1.61 \\
\hline Equality-test & Mean & Std. dev. & Mean & Std. dev. & Mean & Std. dev. \\
P-value & 0.00 & 0.00 & 0.23 & 0.1 & 0.00 & 0.43
\end{tabular}

Table 3: Counter-flow experiment. Mean, standard deviation (in seconds) and p-value of the equality-tests for the travel time in the data and for the simulation. The number of observations is $N=300$.

\subsection{Cross-flow experiment}

To further investigate the model behaviour, an orthogonal cross-flow experiment is considered. The corresponding data has been collected by Plaue et al. (2012), and involves two groups of pedestrians consisting of 78 and 143 students, respectively. The dimensions of the experimental set-up are shown in Fig. 20. The layout is approximated by hexagons of size $a=1.2 \mathrm{~m}$. Pedestrian trajectories are available only within a $3 \mathrm{~m}$-by- $3 \mathrm{~m}$ area where the two groups cross, as indicated by the hatched area. The same experiment has been analysed with a microscopic cellular automata model by Crociani and Lämmel (2016), which is used as a qualitative benchmark.

Fig. 21 compares the empirically observed sequences of density and mean speed with simulation results obtained by the mesoscopic model proposed in this work, and by the aforementioned CA model. Mean value and standard deviation sequences are averaged over 50 independent simulations, and as before, three different values of the friction parameter are considered, namely $p_{0}=0.2,0.5$ and 1 .

As in previous considerations, best results are obtained with a friction of $p_{0}=0.5$. For that parametriza- 


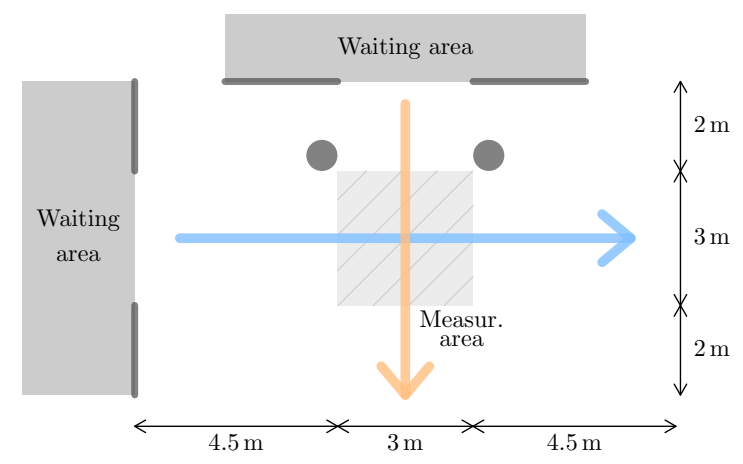

Figure 20: Space configuration of the experiment with crossing flows.

tion, a good agreement with data and the microscopic cellular automaton results. At the onset of the experiment, an underestimation of speed may be perceived, amounting to $v_{0}=1.14 \mathrm{~m} / \mathrm{s}$ instead of the experimentally observed $1.5 \mathrm{~m} / \mathrm{s}$. This could likely be improved by using a more elaborate specification of the fundamental density-speed relation. Overall, we consider promising the fact that the proposed mesoscopic model yields a comparable performance as a microscopic model.
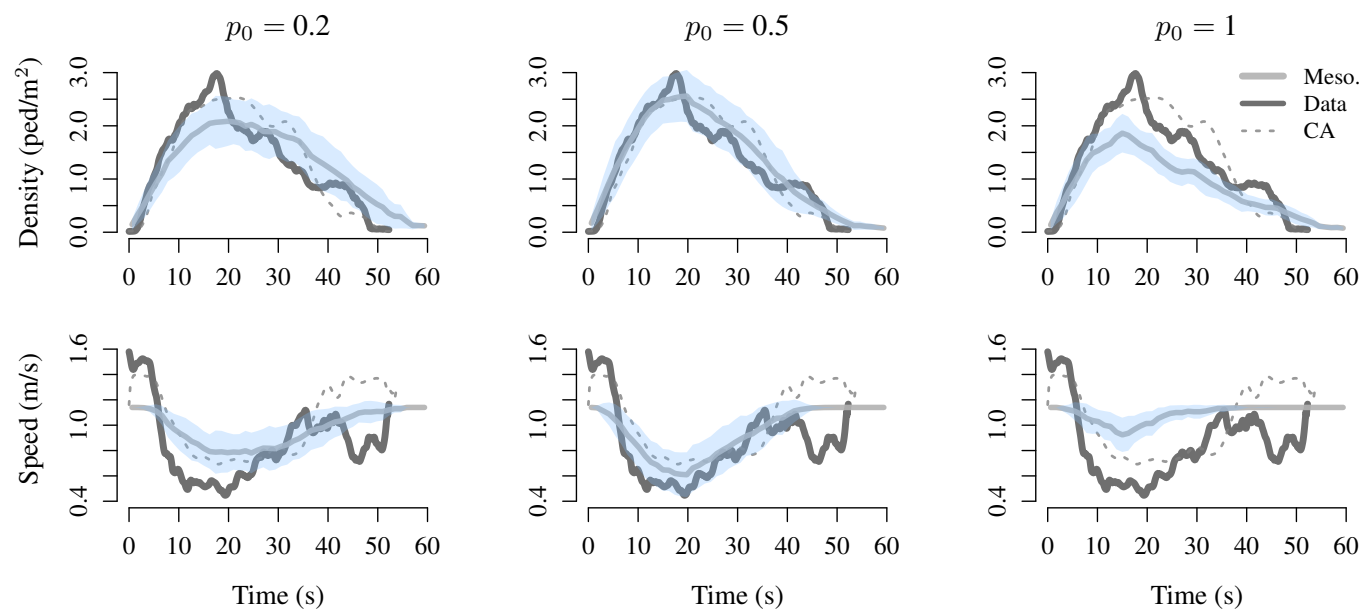

Figure 21: Comparison of measured density and speed sequences to simulation results of the mesoscopic model and of the microscopic CA model introduced by Crociani and Lämmel (2016). Top panels: Density sequences. Bottom panels: Mean speed sequences. From left to right: $p_{0}=0.2, p_{0}=0.5$ and $p_{0}=1$. Friction parameter $p_{0}=0.5$ yields good agreement with the data and the CA model.

\section{Large-scale simulation}

To show the scalability of the proposed model, a large-scale experiment is performed on a real-world scenario involving the Indonesian city of Padang. At a distance of approximately $300 \mathrm{~km}$ to the Great Sumatra fault, Padang is prone to earthquakes and subsequent tsunamis. The city has more than one million inhabitants, and spreads over a low laying area along the shore. Thus, a comprehensive evacuation planning for the so called "last-mile" is essential. The evacuation area is defined as the city's area with an elevation of $10 \mathrm{~m}$ or less, with a size of roughly $7 \times 4 \mathrm{~km}$. The affected population comprises approximately 330,000 individuals who evacuate by foot. The size of the scenario is typically too large to be simulated with a microscopic model. A corresponding in-depth analysis and planning has been done in the "Last-Mile" project (see e.g. Taubenböck et al.; 2013). Part of the project deals with the simulation of the evacuation itself, using a mesoscopic agentbased queue simulation model (Lämmel et al.; 2009), which is part of MATSim (Horni et al.; 2016). In the following, the Padang evacuation scenario is considered to benchmark the calibrated version of the proposed mesoscopic model against the aforementioned large-scale agent-based simulator. 
The queue model by Lämmel et al. (2009) considers link-wise flow capacity restrictions, and exhibits a trapezoidal fundamental diagram with a capacity regime that reaches from medium densities to almost jam density. This stands in contrast to real-world observations, resembling rather triangular-shaped fundamental diagrams. Consequently, the queue model is known to overestimate the flow at high densities, which might lead to a high overall throughput and thus unrealistically short evacuation times.

Evacuation planning usually requires the application of routing strategies. Routes are best represented on a graph-like structure. Thus the proposed model has to be mapped on a graph. An obvious approach is to map the intersections of the street network to nodes and the street segments in between intersections to links. In principle, one could overlay the street network onto a hexagonal grid of the size of the network's bounding box and then "punch out" the walkable area. However, by doing so the hexagonal grid would not be aligned with the links and thus two links with the same physical dimension but different alignment might display different flow dynamics. In order to avoid such artefacts, the hexagonal grid is modelled individually for each link, i.e., the cells are always aligned with the underlying link. The nodes of the network are modelled as irregular cells with an area proportional to the width of the adjacent links as illustrated in Fig. 22. The faces of such node cells are connected to the cells at the boundary (green cells in Fig. 22) of the adjacent links. A map section of the city and an illustration of the mapping to the network model is depicted in Fig. 23. The overall network consists of 6289 nodes and 16978 links.

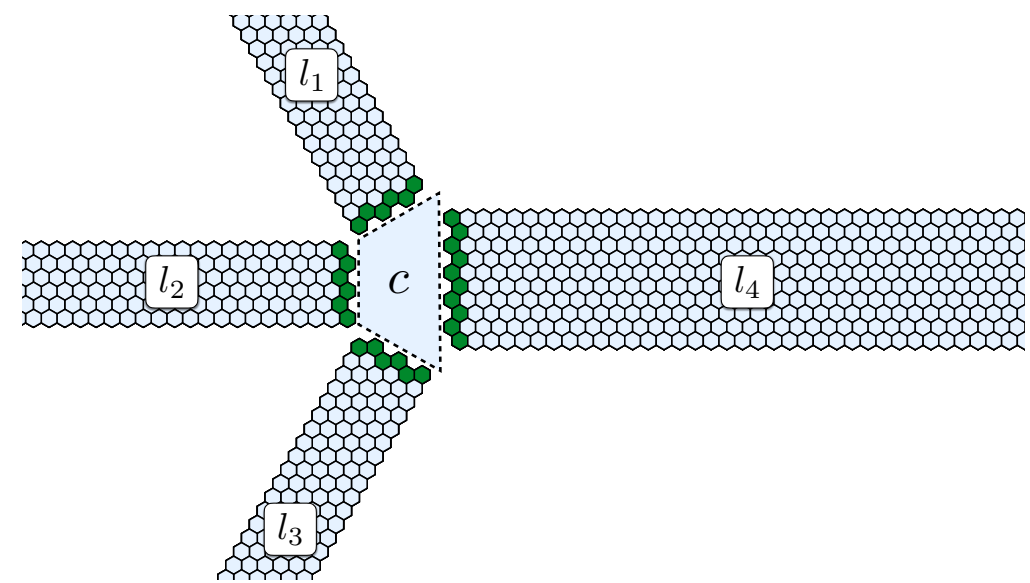

Figure 22: Illustration of the network model. The three links $\left(l_{1}, l_{2}, l_{3}\right)$ are connected via the irregular cell $c$.

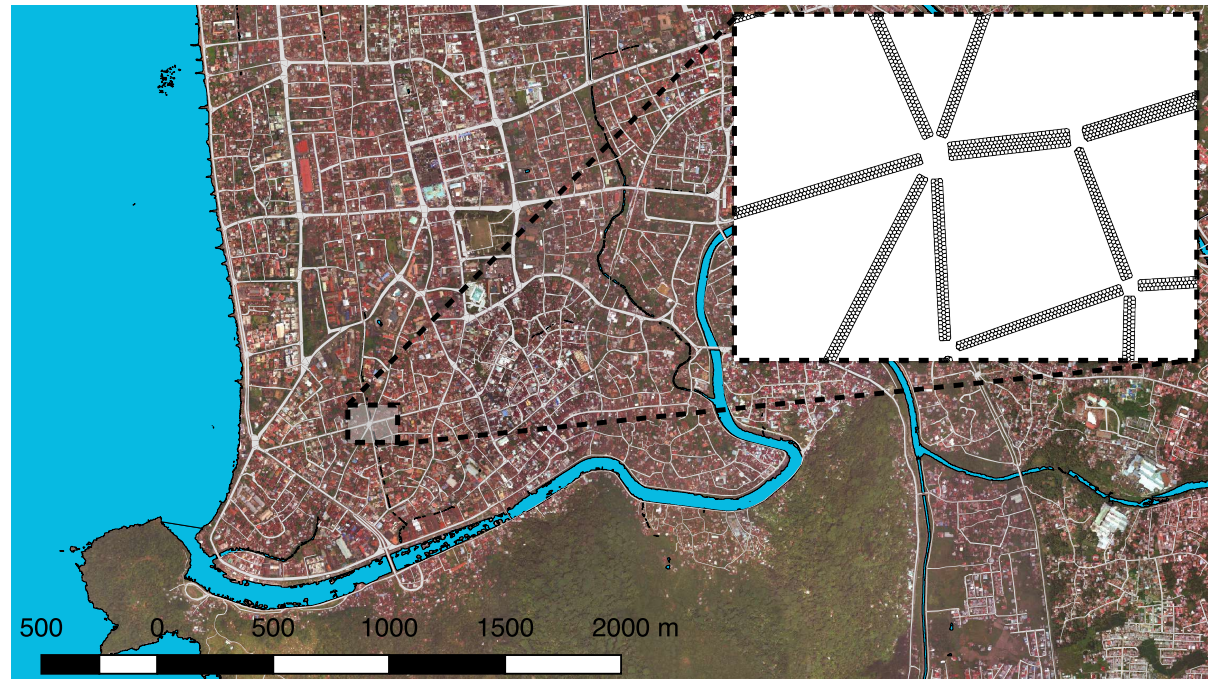

Figure 23: Large-scale scenario. Map section of the evacuation area and an illustration how the streets are mapped to the network model. 
As the number of pedestrians and size of the area are fixed, the computational burden largely depends on the number of jumps each pedestrian performs on their journey. The number of required jumps is proportional to the number of hexagonal cells. Thus, reducing the number of cells (by increasing their size) will reduce the computational burden. As it has been shown in Fig. 7, the cell size (with $a \geq 1 \mathrm{~m}$ ) has no substantial impact on unidirectional flow, as it is prevalent during evacuation. To ensure that the simulated pedestrians are able to reach their desired speed $v_{0}$, it is required that

$$
N \geq 1+\frac{v_{0}}{\gamma}
$$

where $N$ is the maximum number of pedestrians per cell. Based on Eq. (16), a simulation environment consisting of 935,888 hexagonal cells is obtained.

It is assumed that pedestrians evacuate to the closest safe destination, pursuing the shortest path in time according to a Nash equilibrium (Nash; 1951). For a discussion of the Nash equilibrium in the context of evacuation, we refer the interested reader to Lämmel (2011). Simulation results are provided in Fig. 24, showing the cumulative arrivals of evacuees over time.

The average evacuation time for the queue model and the present model amount to 31:34 min and 35:24 min, respectively. The overall dynamics of the evacuation process are similar, as can be perceived by the almost linear slope in Fig. 24. These findings are in keeping with expectations. Reasons for the marginally lower evacuation speed in the stochastic model include differences in the underlying flow dynamics that are arguably more accurate (as discussed at the beginning of this section), as well as the fact that it has been calibrated based on laboratory experiments (see Sec. 5.1). In the case of an actual evacuation, at least free-flow speeds would likely be larger than under experimental conditions. In fact, in the queue-model a free-flow speed of $1.66 \mathrm{~m} / \mathrm{s}$ has been assumed, in contrast to only $1.14 \mathrm{~m} / \mathrm{s}$ in this work.

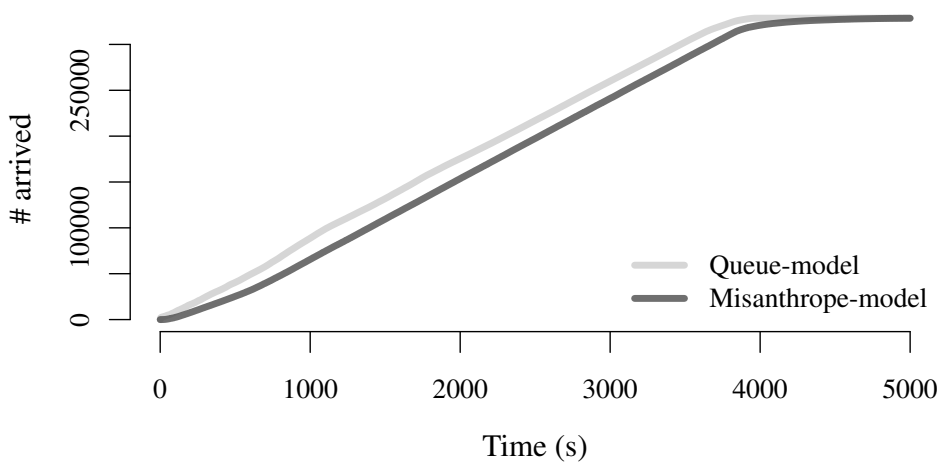

Figure 24: Evacuation times in the large-scale experiment. Evacuation curves as obtained with the present and the queue simulation model (Lämmel; 2011, Chapter 4).

A single iteration of the Padang scenario can be computed faster than real-time with both models ${ }^{1}$. The actual computation time varies depending on the computer architecture. The queue model is roughly one order of magnitude faster. In return, the dynamics provided by the present model are substantially richer and presumably more realistic, while still being efficient enough to consider large-scale scenarios.

\section{Conclusion}

The proposed pedestrian flow model is one of only few mesoscopic models for describing pedestrian dynamics in two dimensions. Most importantly, unlike point queue models, our model can describe space utilization and volume exclusion of pedestrians explicitly. It can inherently reproduce stochasticity and, in principle, population heterogeneity in terms of individual walking behavior, which both are important advantages over existing macroscopic models. Compared to microscopic approaches such as the Social Force model, it typically incurs lower computational costs, making it applicable to large-scale problems. Moreover, the number of parameters is, depending on the specification, lower, allowing for an effective calibration on real data. Even when using a minimal model specification, the model is capable of reproducing various key phenomena of pedestrian dynamics, such as shock- and rarefaction waves in presence of obstacles, or lane formation

\footnotetext{
${ }^{1}$ The approximation of a Nash equilibrium usually requires several hundred iterations.
} 
in counter-flow. A comparison of model predictions with empirical data yields for various experiments a good agreement, both in absolute terms and if contrasted with a related microscopic modelling approach.

Various possibilities for further improvement exist. More elaborate specifications of the friction in the supply function, or of the density-speed relationship (e.g. to capture anisotropy Hänseler, Lam, Bierlaire, Lederrey and Nikolić; 2017) can be envisaged. More importantly, the presented model can be combined with an en-route path choice model to explicitly model the desired walking direction, instead of considering it as an exogenous parameter. We leave this for future research.

\section{Acknowledgements}

This work is supported by the German Research Foundation DFG (Deutsche Forschungsgemeinschaft Grant number SE 1789/4-1).

\section{References}

Abdelghany, A., Abdelghany, K., Mahmassani, H. S. and Al-Zahrani, A. (2012). Dynamic simulation assignment model for pedestrian movements in crowded networks, Transportation Research Record: Journal of the Transportation Research Board 2316(1): 95-105.

Al-Gadhi, S. A. H. and Mahmassani, H. S. (1991). Simulation of crowd behavior and movement: Fundamental relations and application, Transportation Research Record: Journal of the Transportation Research Board 1320(1320): 260268.

Antonini, G., Bierlaire, M. and Weber, M. (2006). Discrete choice models of pedestrian walking behavior, Transportation Research Part B: Methodological 40(8): 667-687.

Asano, M., Sumalee, A., Kuwahara, M. and Tanaka, S. (2007). Dynamic cell transmission-based pedestrian model with multidirectional flows and strategic route choices, Transportation Research Record: Journal of the Transportation Research Board 2039: 42-49.

Bellomo, N. and Dogbe, C. (2011). On the modeling of traffic and crowds: A survey of models, speculations, and perspectives, SIAM Review 53(3): 409-463.

Blue, V. J. and Adler, J. L. (2001). Cellular automata microsimulation for modeling bi-directional pedestrian walkways, Transportation Research Part B: Methodological 35(3): 293-312.

Boltes, M., Seyfried, A., Steffen, B. and Schadschneider, A. (2010). Automatic extraction of pedestrian trajectories from video recordings, Pedestrian and Evacuation Dynamics, Springer, Berlin Heidelberg, pp. 43-54.

Borgers, A. and Timmermans, H. (1986). A model of pedestrian route choice and demand for retail facilities within inner-city shopping areas, Geographical analysis 18(2): 115-128.

Burstedde, C., Klauck, K., Schadschneider, A. and Zittartz, J. (2001). Simulation of pedestrian dynamics using a twodimensional cellular automaton, Physica A: Statistical Mechanics and its Applications 295(3): 507-525.

Cocozza-Thivent, C. (1985). Processus des misanthropes, Zeitschrift für Wahrscheinlichkeitstheorie und verwandte Gebiete 70(4): 509-523.

Cocozza-Thivent, C. (1997). Processus stochastiques et fiabilité des systèmes, Springer.

Colombo, R. M. and Rosini, M. D. (2005). Pedestrian flows and non-classical shocks, Mathematical Methods in the Applied Sciences 28(13): 1553-1567.

Corbetta, A., Muntean, A. and Vafayi, K. (2015). Parameter estimation of social forces in pedestrian dynamics models via a probabilistic method, Mathematical Biosciences and Engineering 12(2): 337-356.

Courant, R., Friedrichs, K. and Lewy, H. (1928). Über die partiellen Differenzengleichungen der mathematischen Physik, Mathematische Annalen 100: 32-74.

Crociani, L. and Lämmel, G. (2016). Multidestination pedestrian flows in equilibrium: A cellular automaton-based approach, Computer-Aided Civil and Infrastructure Engineering 31(6): 432-448.

Crociani, L., Lämmel, G. and Vizzari, G. (2016). Multi-scale simulation for crowd management: A case study in an urban scenario, Autonomous Agents and Multiagent Systems, Springer International Publishing, pp. 147-162.

Daamen, W. (2004). Modelling passenger flows in public transport facilities, PhD thesis, Delft University of Technology. 
Daganzo, C. (1994). The cell transmission model: A dynamic representation of highway traffic consistent with the hydrodynamic theory, Transportation Research Part B: Methodological 28(4): 269-287.

Davidich, M. and Köster, G. (2013). Predicting pedestrian flow: A methodology and a proof of concept based on real-life data, PLOS ONE 8(12): 1-11.

Duives, D. C., Daamen, W. and Hoogendoorn, S. P. (2013). State-of-the-art crowd motion simulation models, Transportation Research Part C: Emerging Technologies 37(12): 193-209.

Evans, M. and Hanney, T. (2005). Nonequilibrium statistical mechanics of the zero-range process and related models, Journal of Physics A: Mathematical and Theoretical 38: 195-240.

Eymard, R., Roussignol, M. and Tordeux, A. (2012). Convergence of a misanthrope process to the entropy solution of 1D problems, Stochastic Processes and their Applications 122(11): 3648-3679.

Flötteröd, G. and Lämmel, G. (2015). Bidirectional pedestrian fundamental diagram, Transportation Research Part B: Methodological 71: 194-212.

Fruin, J. J. (1971). Pedestrian planning and design, Metropolitan Association of Urban Designers and Environmental Planners, Michigan, USA.

Gipps, P. G. (1986). Simulation of pedestrian traffic in buildings, Technical Report 35, Institut für Verkehrswesen Universität (TH) Karlsruhe.

Guo, R. Y., Huang, H. J. and Wong, S. C. (2011). Collection, spillback, and dissipation in pedestrian evacuation: A network-based method, Transportation Research Part B: Methodological 45(3): 490-506.

Hall, E. T., Birdwhistell, R. L., Bock, B., Bohannan, P., Diebold, A. R., Durbin, M., Edmonson, M. S., Fischer, J. L., Hymes, D., Kimball, S. T., Barre, W. L., Frank Lynch, S. J., McClellan, J. E., Marshall, D. S., Milner, G. B., Sarles, H. B., Trager, G. L. and Vayda, A. P. (1968). Proxemics [and comments and replies], Current Anthropology 9(2/3): 83108.

Hankin, B. and Wright, R. (1958). Passenger flow in subways, Operational Research Quarterly 9(2): 81-88.

Hänseler, F. S. (2016). Modeling and estimation of pedestrian flows in train stations, PhD thesis, Ecole Polytechnique Fédérale de Lausanne.

Hänseler, F. S., Bierlaire, M., Farooq, B. and Mühlematter, T. (2014). A macroscopic loading model for time-varying pedestrian flows in public walking areas, Transportation Research Part B: Methodological 69: 60-80.

Hänseler, F. S., Lam, W. H. K., Bierlaire, M., Lederrey, G. and Nikolić, M. (2017). A dynamic network loading model for anisotropic and congested pedestrian flows, Transportation Research Part B: Methodological 95: 149-168.

Hänseler, F. S., Molyneaux, N. A. and Bierlaire, M. (2017). Estimation of pedestrian origin-destination demand in train stations, Transportation Science 51(3): 981-997.

Hartmann, D. (2010). Adaptive pedestrian dynamics based on geodesics, New Journal of Physics 12(4): 043032.

Helbing, D., Farkas, I. J. and Vicsek, T. (2000a). Simulating dynamical features of escape panic, Nature 407(487-490).

Helbing, D., Farkas, I., Molnar, P. and Vicsek, T. (2002). Simulation of pedestrian crowds in normal and evacuation situations, Pedestrian and evacuation dynamics 21.

Helbing, D., Farkas, I. and Vicsek, T. (2000b). Freezing by heating in a driven mesoscopic system, Physical Review Letter 84: 1240-1243.

Helbing, D. and Molnár, P. (1995). Social force model for pedestrian dynamics, Physical Review E 51(5): 4282-4286.

Helbing, D., Molnar, P., Farkas, I. J. and Bolay, K. (2001). Self-organizing pedestrian movement, Environment and Planning B 28(3): 361-384.

Hoogendoorn, S. P. and Bovy, P. H. L. (2004). Pedestrian route-choice and activity scheduling theory and models, Transportation Research Part B: Methodological 38(2): 169-190.

Hoogendoorn, S. P. and Daamen, W. (2009). A novel calibration approach of microscopic pedestrian models, Pedestrian behavior: models, data collection and applications p. 195.

Hoogendoorn, S. P., van Wageningen-Kessels, F. L. M., Daamen, W. and Duives, D. C. (2014). Continuum modelling of pedestrian flows: From microscopic principles to self-organised macroscopic phenomena, Physica A: Statistical Mechanics and its Applications 416: 684-694.

Horni, A., Nagel, K. and Axhausen, K. (2016). The Multi-Agent Transport Simulation MATSim, London: Ubiquity Press. 
Huang, L., Wong, S. C., Zhang, M., Shu, C. W. and Lam, W. H. K. (2009). Revisiting Hughes' dynamic continuum model for pedestrian flow and the development of an efficient solution algorithm, Transportation Research Part B: Methodological 43(1): 127-141.

Hughes, R. L. (2002). A continuum theory for the flow of pedestrians, Transportation Research Part B: Methodological 36(6): 507-535

Jabari, S., Zheng, J. and Liu, H. (2014). A probabilistic stationary speeddensity relation based on newells simplified car-following model, Transportation Research Part B: Methodological 68: 205-223.

Johansson, A., Helbing, D. and Shukla, P. (2007). Specification of the social force pedestrian model by evolutionary adjustment to video tracking data, Advances in Complex Systems 10: 271-288.

Kanai, M. (2010). Two-lane traffic-flow model with an exact steady-state solution, Physical Review E 82(6): 066107.

Kirchner, A. and Schadschneider, A. (2002). Simulation of evacuation processes using a bionics-inspired cellular automaton model for pedestrian dynamics, Physica A: Statistical Mechanics and its Applications 312(1): 260-276.

Lämmel, G. (2011). Escaping the Tsunami: Evacuation Strategies for Large Urban Areas. Concepts and Implementation of a Multi-Agent Based Approach, PhD thesis, Technische Universität Berlin.

Lämmel, G., Grether, D. and Nagel, K. (2010). The representation and implementation of time-dependent inundation in large-scale microscopic evacuation simulations, Transportation Research Part C: Emerging Technologies 18(1): 8498.

Lämmel, G., Klüpfel, H. and Nagel, K. (2009). The MATSim network flow model for traffic simulation adapted to largescale emergency egress and an application to the evacuation of the Indonesian city of Padang in case of a tsunami warning, in H. Timmermans (ed.), Pedestrian Behavior, Emerald Group Publishing Limited, chapter 11, pp. 245-265.

Lämmel, G., Seyfried, A. and Steffen, B. (2014). Large-scale and microscopic: a fast simulation approach for urban areas, Transportation Research Board Annual Meeting, pp. 14-3890.

Lebacque, J.-P. (1996). The godunov scheme and what it means for first order traffic flow models, Proceeding of the 13th International Symposium on Transportation and Traffic Theory, Pergamon Press, Oxford, pp. 647-677.

Liggett, T. (1985). Interacting particle systems, Springer.

Løvås, G. G. (1994). Modeling and simulation of pedestrian traffic flow, Transportation Research Part B: Methodological 28(6): 429-443.

Nash, J. (1951). Non-cooperative games, The Annals of Mathematics 54(2): 286-295.

Newell, G. (2002). A simplified car-following theory: a lower order model, Transportation Research Part B: Methodological 36(3): 195-205.

Nikolić, M., Bierlaire, M., Farooq, B. and de Lapparent, M. (2016). Probabilistic speed-density relationship for pedestrian traffic, Transportation Research Part B: Methodological 89: 58-81.

Ondřej, J., Pettré, J., Olivier, A.-H. and Donikian, S. (2010). A synthetic-vision based steering approach for crowd simulation, ACM Transactions on Graphics, Vol. 29, ACM, p. 123.

Osorio, C., Flötteröd, G. and Bierlaire, M. (2011). Dynamic network loading: A stochastic differentiable model that derives link state distributions, Transportation Research Part B: Methodological 45(9): 1410-1423.

Paris, S., Pettré, J. and Donikian, S. (2007). Pedestrian reactive navigation for crowd simulation: a predictive approach, Computer Graphics Forum, Vol. 26, Wiley Online Library, pp. 665-674.

Piccoli, B. and Tosin, A. (2011). Time-evolving measures and macroscopic modeling of pedestrian flow, Archive for Rational Mechanics and Analysis 199(3): 707-738.

Plaue, M., Chen, M., Bärwolff, G. and Schwandt, H. (2012). Multi-view extraction of dynamic pedestrian density fields, Photogrammetrie - Fernerkundung - Geoinformation 2012(5): 547-555.

Schadschneider, A., Chowdhury, D. and Nishinari, K. (2010). Stochastic Transport in Complex Systems. From Molecules to Vehicles, Elsevier Science Publishing Co Inc.

Seer, S., Bauer, D., Brandle, N. and Ray, M. (2008). Estimating pedestrian movement characteristics for crowd control at public transport facilities, Intelligent Transportation Systems, IEEE, pp. 742-747.

Seyfried, A., Steffen, B., Klingsch, W. and Boltes, M. (2005). The fundamental diagram of pedestrian movement revisited, Journal of Statistical Mechanics: Theory and Experiment 2005(10): P10002. 
Shende, A., Singh, M. P. and Kachroo, P. (2011). Optimization-based feedback control for pedestrian evacuation from an exit corridor, IEEE Transactions on Intelligent Transportation Systems 12(4): 1167-1176.

Spitzer, F. (1970). Interaction of Markov processes, Advances in Mathematics 5(2): 246-290.

Steffen, B. and Chraibi, M. (2014). Multiscale Simulation of Pedestrians for Faster Than Real Time Modeling in Large Events, Springer International Publishing, Cham, pp. 492-500.

Steffen, B. and Seyfried, A. (2010). Methods for measuring pedestrian density, flow, speed and direction with minimal scatter, Physica A: Statistical Mechanics and its Applications 389(9): 1902-1910.

Taubenböck, H., Goseberg, N., Lämmel, G., Setiadi, N., Schlurmann, T., Nagel, K., Siegert, F., Birkmann, J., Traub, K.-P., Dech, S., Keuck, V., Lehmann, F., Strunz, G. and Klüpfel, H. (2013). Risk reduction at the "Last-Mile": an attempt to turn science into action by the example of Padang, Indonesia, Natural Hazards 65(1): 915-1945.

Tordeux, A., Roussignol, M., Lebacque, J.-P. and Lassarre, S. (2014). A stochastic jump process applied to traffic flow modelling, Transpormetrica 10(4): 350-375.

Treiber, M. and Kesting, A. (2013). Traffic Flow Dynamics, Springer, Berlin.

Treuille, A., Cooper, S. and Popović, Z. (2006). Continuum crowds, ACM Transactions on Graphics (TOG), Vol. 25, ACM, pp. 1160-1168.

Weidmann, U. (1992). Transporttechnik der Fußgänger, Schriftenreihe des IVT Nr. 90, Institute for Transport Planning and Systems, ETH Zürich, Switzerland.

Wilensky, U. (1999). Netlogo, http://ccl.northwestern.edu/netlogo/, Center for Connected Learning and Computer-Based Modeling, Northwestern University, Evanston, IL.

URL: http://ccl.northwestern.edu/netlogo/

Wong, S., Leung, W., Chan, S., William, H., Nelson, H., Yung, H., Liu, C. and Zhang, P. (2010). Bidirectional pedestrian stream model with oblique intersecting angle, Journal of Transportation Engineering 136(3): 234-242.

Xie, S. and Wong, S. (2015). A bayesian inference approach to the development of a multidirectional pedestrian stream model, Transportmetrica A: Transport Science 11(1): 61-73.

Zhang, J., Klingsch, W., Schadschneider, A. and Seyfried, A. (2012). Ordering in bidirectional pedestrian flows and its influence on the fundamental diagram, Journal of Statistical Mechanics: Theory and Experiment 2012(02): P02002.

\section{Appendix: Computational complexity of the model}

The calculation of the jump rate $b$ involves the computation of flows $J$, see Eqs. (3) and (7), and of destination factors $D$, see Eq. (4). The calculation of $J$ requires to determine the pedestrian class $c$ and a few operations with four comparisons. This represents approximately $C_{J}=10$ operations. The identification of the desired direction requires the calculation of a cosine within the weight function $\omega$, and the flow for each of the accessible neighbours, i.e. at most 6 . The computation of a cosine requires approximately $C_{D}=35$ operations. The calculation may be reduced by storing the weight in a look-up table. In total, the computation of the jump rate $b$ requires about $C_{b}=6\left(C_{J}+C_{D}\right)$ operations per class.

After the calculation of the jump rate, the time for the next jump is computed from a pseudo-random variable generator. For an exponential distribution, this takes about $C_{r}=40$ operations. The jump times are ordered in a list to successively get the minimal times. With standard sorting, it requires about $5 \ln (K d s)$ operations to find the position, and about $K d s / 3$ operations to shift the tail of the event list, $K$ being the number of cells and $d$ the number of desired directions among the pedestrians and $s$ the number of classes. Therefore the event-based update needs $C_{e}=$ $5 \ln (K d s)+K d s / 3$ operations per jump. For large systems, this will be the dominant load. In the worst case, 10 neighbours have to be updated at each jump and all the $d$ desired destinations are systematically affected. In such a case, a jump needs $10 d\left(6\left(C_{J}+C_{D}\right)+C_{r}+C_{e}\right)$ operations per class.

The frequency of the jumps depends on the jump rates which depend themselves on the density through the fundamental diagram $J_{\rho}$. The complexity by unit of time for the simulation of the model is hence

$$
C(\rho, K, d, s)=10 K d s \kappa J_{\rho}\left(C_{b}+C_{r}+5 \ln (K d s)+K d s / 3\right) .
$$

The capacity of current portable computers is about $6 \cdot 10^{8}$ operations per second. If we consider a single pedestrian class in an evacuation scenario for which $d=1$ and that hexagonal cells have size $a=2.5 \mathrm{~m}$ then the model can be simulated in real time for at least $K=5000$. Such systems represent respectively about 150,000 pedestrians at a critical density level where the flow is maximal, and can contain up to 500,000 pedestrians at jam density.

Inserting new jump times in the ordered list of events is the most costly part of the procedure when the size of the system is large. Yet it can be speeded up. Simply having an ordered list of events and inserting a new event at the proper place requires a number of operations proportional to the the number of cells $K$. Organising the event list as a self 
balancing binary tree gives a complexity of $O(\ln K)$ for the insertion of a new event as well as for finding the next event to treat. In such a case, a system with $K$ cells and mean density level $\rho$ has the complexity of $O\left(J_{\rho} K \ln K\right), J_{\rho}$ being the mean flow. The fastest microscopic pedestrian model known to the authors is a cellular automata model (CA) with random sequential ordering (Steffen and Chraibi; 2014) which has a complexity of $O(\rho K \ln (\rho K)$. For a macroscopic model, the number of calculations per unit of time depends on the number of cells and does not depend on the density. The complexity is $O(K)$.

A comparative experiment is carried out with a microscopic model (namely the totally asymmetric exclusion process (TASEP) with sequential update (Liggett; 1985; Steffen and Chraibi; 2014)), the mesoscopic approach, and the cell transmission (CT) macroscopic model (Daganzo; 1994). A periodic uni-dimensional system that can contain up to 5,000 pedestrians is simulated during time $T=3,600$. The time step in the microscopic and macroscopic model are set to the unit while the cell in the mesoscopic and macroscopic models can contain up to 40 pedestrians. The models are all based on the identical triangular fundamental diagram $J(x)=\min \{x, 1-x\}$, with $x \in[0,1]$ the concentration. The simulations are done with the software NetLogo (Wilensky; 1999) on a $2.7 \mathrm{GHz}$ processor.

The computation times according to the concentration are presented in Fig. 25. As expected, the simulation time monotonically increases with the density for the microscopic model, while it is proportional to the flow for the continuous time approach and constant for the macroscopic model. The mesoscopic model is more efficient than the microscopic one for any density level, and than the macroscopic model for low and high density levels. In the worst case (at the critical density level), the mesoscopic model is approximately three times slower than the macroscopic model.

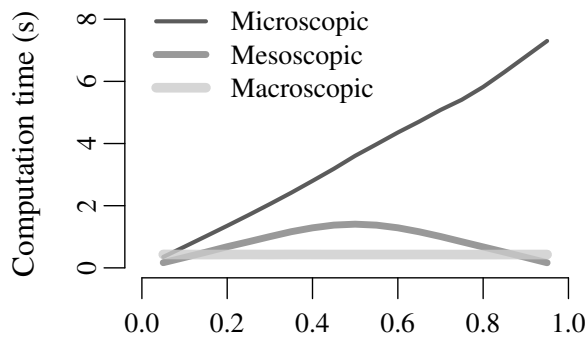

Concentration

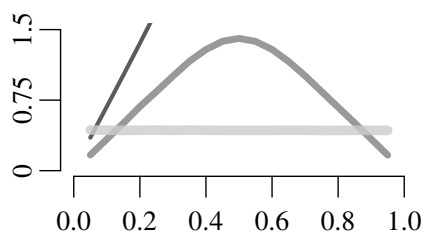

Concentration

Figure 25: Computation times for the simulation of the periodic uni-dimensional system during a simulation $T=3,600$ with the microscopic TASEP model, the mesoscopic approach and the CT macroscopic model. 